\title{
DIGITALCOMMONS
}

@WAYNESTATE -

Wayne State University

$12-4-2003$

\section{Necessary Conditions in Nonsmooth Minimization Via Lower and Upper Subgradients}

Boris S. Mordukhovich

Wayne State University, boris@math.wayne.edu

\section{Recommended Citation}

Mordukhovich, Boris S., "Necessary Conditions in Nonsmooth Minimization Via Lower and Upper Subgradients" (2003).

Mathematics Research Reports. Paper 20.

http://digitalcommons.wayne.edu/math_reports/20

This Technical Report is brought to you for free and open access by the Mathematics at DigitalCommons@WayneState. It has been accepted for inclusion in Mathematics Research Reports by an authorized administrator of DigitalCommons@WayneState. 


\section{NECESSARY CONDITIONS IN NONSMOOTH MINIMIZATION VIA LOWER AND UPPER SUBGRADIENTS}

BORIS S. MORDUKHOVICH

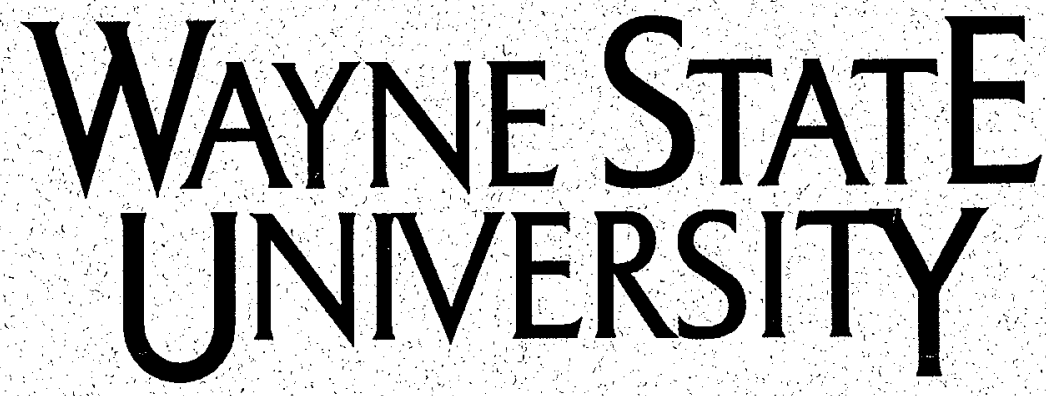

Detroit, MI 48202

Department of Mathematics

Research Report

2003 Series

\#16 


\title{
NECESSARY CONDITIONS IN NONSMOOTH MINIMIZATION VIA LOWER AND UPPER SUBGRADIENTS
}

\author{
BORIS S. MORDUKHOVICH ${ }^{1}$ \\ Department of Mathematics, Wayne State University \\ Detroit, MI 48202, U.S.A.
}

\begin{abstract}
The paper concerns first-order necessary optimality conditions for problems of minimizing nonsmooth functions under various constraints in infinite-dimensional spaces. Based on advanced tools of variational analysis and generalized differential calculus, we derive general results of two independent types called lower subdifferential and upper subdifferential optimality conditions. The former ones involve basic/limiting subgradients of cost functions, while the latter conditions are expressed via Fréchet/regular upper subgradients in fairly general settings. All the upper subdifferential and major lower subdifferential optimality conditions obtained in the paper are new even in finite dimensions. We give applications of general optimality conditions to mathematical programs with equilibrium constraints deriving new results for this important class of intrinsically nonsmooth optimization problems.
\end{abstract}

Mathematics Subject Classifications (2000): 49J52, 49K27, 90C48.

Key words: variational analysis, nonsmooth optimization, generalized differentiation, lower and upper subgradients, infinite-dimensional spaces, necessary optimality conditions, mathematical programs with equilibrium constraints.

\section{Introduction}

This paper is devoted to the study of necessary optimality conditions for constrained minimization problems in infinite-dimensional spaces. A general problem of this type with (non-specified) geometric constraints can be written as:

$$
\text { minimize } \varphi_{0}(x) \text { subject to } x \in \Omega \subset X \text {, }
$$

where $\varphi_{0}: X \rightarrow \bar{R}:=[-\infty, \infty]$ is an extended-real-valued function on a Banach space $X$ finite at a reference point, and where $\Omega$ is an arbitrary nonempty subset of $X$. The constrained problem (1.1) is obviously equivalent to the unconstrained problem:

$$
\operatorname{minimize} \varphi_{0}(x)+\delta(x ; \Omega), \quad x \in X
$$

where the indicator function $\delta(\cdot ; \Omega)$ of $\Omega$, defined by $\delta(x ; \Omega):=0$ if $x \in \Omega$ and $\delta(x ; \Omega):=\infty$ otherwise, imposes an "infinite penalty" on the constraint violation. Denoting by

$$
\widehat{\partial} \varphi(\bar{x}):=\left\{x^{*} \in X^{*} \mid \liminf _{x \rightarrow \bar{x}} \frac{\varphi(x)-\varphi(\bar{x})-\left\langle x^{*}, x-\bar{x}\right\rangle}{\|x-\bar{x}\|} \geq 0\right\}
$$

\footnotetext{
${ }^{1}$ Research was partly supported by the National Science Foundation under grants DMS-0072179 and DMS-0304989.
} 
the (lower) Fréchet subdifferential, known also as the regular or viscosity subdifferential, of $\varphi: X \rightarrow$ $\widetilde{R}$ at $\bar{x}$ with $|\varphi(\bar{x})|<\infty$, one can observe directly from the definition that the following generalized Fermat rule holds: if $\bar{x}$ a local minimizer of $\varphi$, then $0 \in \widehat{\partial} \varphi(\bar{x})$. Applying this to the unconstrained form of (1.1), we get

$$
0 \in \widehat{\partial}\left(\varphi_{0}+\delta(\cdot ; \Omega)\right)(\bar{x})
$$

provided that $\bar{x}$ gives a local minimum to $\varphi_{0}(x)$ subject to $x \in \Omega$. If $\varphi_{0}$ happens to be Fréchet differentiable at $\bar{x}$ with the derivative/gradient $\nabla \varphi_{0}(\bar{x})$, then (1.3) is equivalent to the inclusion

$$
-\nabla \varphi_{0}(\bar{x}) \in \widehat{N}(\bar{x} ; \Omega):=\left\{x^{*} \in X^{*} \mid \underset{\substack{\Omega \\ x \rightarrow \bar{x}}}{\limsup } \frac{\left\langle x^{*}, x-\bar{x}\right\rangle}{\|x-\bar{x}\|} \leq 0\right\}
$$

where $\hat{N}(\bar{x} ; \Omega)=\widehat{\partial} \delta(\bar{x} ; \Omega)$ is the Fréchet normal cone to $\Omega$ at $\bar{x} \in \Omega$, and where $x \stackrel{\Omega}{x}$ means that $x \rightarrow \bar{x}$ with $x \in \Omega$. Indeed, the equivalence between (1.3) and (1.4) follows from the simple sum rule for Fréchet subgradients:

$$
\widehat{\partial}\left(\varphi_{0}+\varphi_{1}\right)(\ddot{x})=\nabla \varphi_{0}(\vec{x})+\widehat{\partial} \varphi_{1}(\bar{x})
$$

held in Banach spaces for any function $\varphi_{0}$ Fréchet differentiable at $\bar{x}$ and an arbitrary function $\varphi_{1}: X \rightarrow \bar{R}$ finite at $\bar{x}$.

If $\varphi_{0}$ is not Fréchet differentiable at $\bar{x}$, the above way does not lead to valuable optimality conditions, since Fréchet-like subgradients generally possess a poor calculus even for simple nonsmooth functions in finite dimensions. To be able to proceed further, one needs to employ more robust subdifferentials satisfying required calculus rules. In what follows we are going to develop such an approach based on our basic/sequential limiting subgradients of extended-real-valued functions and the corresponding normal cone and coderivative constructions for sets and set-valued mappings enjoying a number of useful calculus rules in arbitrary Banach spaces and fairly comprehensive calculi in the Asplund space setting; see below. In this way we derive general first-order optimality conditions of the lower subdifferential type for minimization problems with various constraints typically arising in applications. In particular, for problem (1.1), which is actually the simplest albeit general constrained optimization problem, the corresponding lower subdifferential optimality condition reads as

$$
0 \in \partial \varphi_{0}(\tilde{x})+N(\bar{x} ; \Omega)
$$

provided that $\varphi_{0}$ is Lipschitz continuous around $\bar{x}$, as well as under more general qualification and normal compactness assumptions. We also derive lower subdifferential optimality conditions for minimization problems with many geometric constraints given by set intersections, with operator constraints defined by inverse images of set-valued mappings, with functional constraints given by equalities and inequalities, and with equilibrium constraints governed by parametric generalized equations and variational inequalities. For the latter class of minimization problems related to hierarchical optimization, second-order subdifferential constructions are useful in applications to first-order optimality conditions. Note that the realization of this approach in the case of infinitedimensional spaces is based not only on calculus rules for subdifferentials and coderivatives, but also on calculus results ensuring the preservation of the so-called sequential normal compactness 
properties for functions, sets, and set-valued mappings that are automatic in finite dimensions while playing a crucial role in infinite-dimensional optimization and variational analysis.

Along with lower subdifferential optimality conditions held for problems of minimizing general cost functions, we derive necessary optimality conditions of a new type that are especially efficient for special classes of functions under minimization and those describing inequality constraints, being often more powerful for these special classes than the former ones. Such upper subdifferential (or superdifferential) conditions, which seem to be rather surprising for minimization problems, involve the following Fréchet upper subdifferential construction for a given function $\varphi: X \rightarrow \widetilde{\mathbb{R}}$ finite at $\bar{x}$ defined by

$$
\widehat{\partial}^{+} \varphi(\bar{x}):=-\widehat{\partial}(-\varphi)(\bar{x})=\left\{x^{*} \in X^{*} \mid \limsup _{x \rightarrow \bar{x}} \frac{\varphi(x)-\varphi(\bar{x})-\left\langle x^{*}, x-\bar{x}\right\rangle}{\|x-\bar{x}\|} \leq 0\right\} .
$$

Note that the upper subdifferential (1.6) is known also as the "superdifferential" being particularly useful in the theory of viscosity solutions for PDE.problems; see, e.g., [5]. Following [25], we adopt the "upper" terminology, which seems to be more in accordance with the sense of such constructions.

It happens that Fréchet upper subgradients of extended-real-valued functions admit certain smooth variational descriptions allowing us to reduce, in particular, necessary optimality conditions for problem (1.1), given each $x^{*} \in \widehat{\partial}^{+} \varphi_{0}(\bar{x})$, to those for a counterpart of (1.1) with a Fréchet differentiable cost function whose derivative equals $x^{*}$. This leads to upper subdifferential conditions for (1.1) of the type

$$
-\widehat{\partial}^{+} \varphi_{0}(\bar{x}) \subset \hat{N}(\bar{x} ; \Omega)
$$

Such conditions carry nontrivial information for minimization problems with $\widehat{\partial}^{+} \varphi_{0}(\bar{x}) \neq \emptyset$, e.g., for problems of minimizing concave functions or, more generally, for nonsmooth functions with $\widehat{\partial} \varphi_{0}(\bar{x})=\emptyset$. Note that the emptiness of $\widehat{\partial}^{+} \varphi_{0}(\bar{x})$ is itself is an easy checkable necessary condition for minimization of $\varphi_{0}$ that does not depend on constraints.

Upper subdifferential conditions are especially efficient for the class of functions that are upper regular at a local minimum point; see Sections 2 and 3. They are generally independent from lower subdifferential ones but may give essentially stronger results for some classes of minimization problems. In this paper we derive upper subdifferential conditions for minimization problems with the same types of general constraints as the lower subdifferential conditions discussed above. More specific results of the upper subdifferential type are obtained for minimization problems with inequality constraints.

The rest of the paper is organized as follows. Section 2 presents basic definitions and preliminaries from generalized differentiation and variational analysis widely used in what follows. In Section 3 we derive necessary optimality conditions of both lower and upper subdifferential types for constrained minimization problems in form (1.1) and also for problems with many geometric constraints given by set intersections. Section 4 deals with minimization problems that contain, together with geometric constraints, also constraints of operator and functional types given generally by inverse images of set-valued mappings and particularly by equalities and inequalities with real-valued functions. The final Section 5 is devoted to lower and upper subdifferential optimality conditions for general classes of mathematical programs with equilibrium constraints in infinite-dimensional spaces. Most of the results obtained seem to be new not only in the case of 
upper subdifferential conditions but for lower subdifferential ones as well, even in finite dimensions. They admit essential simplifications in finite-dimensional spaces when all the assumptions on the sequential normal compactness hold automatically.

Our notation is basically standard, with special symbols introduced where they are defined. Unless otherwise stated, all spaces considered are Banach whose norms are always denoted by $\|\cdot\|$. For any space $X$ we consider its dual space $X^{*}$ equipped with the weak ${ }^{*}$ topology $w^{*}$, where $\langle\cdot, \cdot \cdot\rangle$ means the canonical pairing. For multifunctions $F: X \rightarrow X^{*}$ the expression

$$
\begin{aligned}
\operatorname{Limsup}_{x \rightarrow \bar{x}} F(x):=\left\{x^{*} \in X^{*} \mid\right. & \exists \text { sequences } x_{k} \rightarrow \bar{x} \text { and } x_{k}^{*} \stackrel{w^{*}}{\rightarrow} x^{*} \\
& \text { with } \left.x_{k}^{*} \in F\left(x_{k}\right) \text { for all } k \in \mathbb{N}\right\}
\end{aligned}
$$

signifies the sequential Painlevé-Kuratowski upper/outer limit with respect to the norm topology in $X$ and the weak ${ }^{*}$ topology in $X^{*}$, where $\mathbb{I N}:=\{1,2, \ldots\}$.

\section{Preliminaries}

As mentioned in Section 1, for applications to necessary optimality conditions in this paper we need, along with the Fréchet-like constructions (1.2), (1.4), and (1.6), their robust counterparts defined as follows. The reader can find more details on these constructions and their history in the books $[12,25]$ and papers $[3,13,18]$ in, respectively, finite and infinite dimensions.

Given a nonempty subset $\Omega$ of a Banach space $X$ and a number $\varepsilon \geq 0$, we first define the $\varepsilon$-enlargement of the cone $\widehat{N}(\cdot ; \Omega)$ in $(1.4)$ by

$$
\widehat{N}_{\varepsilon}(x ; \Omega):=\left\{x^{*} \in X^{*} \mid \underset{\substack{u \rightarrow x \\ u \rightarrow x}}{\limsup } \frac{\left\langle x^{*}, u-x\right\rangle}{\|u-x\|} \leq \varepsilon\right\} \text { for } x \in \Omega
$$

and by $\widehat{N}_{\varepsilon}(x ; \Omega):=\emptyset$ for $x \notin \Omega$. Then the basic normal cone to $\Omega$ at $\bar{x} \in \Omega$ is given by

$$
N(\bar{x} ; \Omega):=\operatorname{Limsup}_{\substack{x \rightarrow \bar{x} \\ \varepsilon \downarrow 0}} \widehat{N}_{\varepsilon}(x ; \Omega)
$$

as the sequential Painlevé-Kuratowski upper limit of $\varepsilon$-normals (2.1) at nearby points. When $X$ is Asplund (i.e., its every separable subspace has a separable dual; see [24] for more information) and $\Omega$ is closed around $\bar{x}$, one can equivalently put $\varepsilon=0$ in $(2.2)$ and hence replace $\widehat{N}_{\varepsilon}(\cdot ; \Omega)$ with the Fréchet normal cone; see [18, Theorem 2.9]. However, one cannot remove $\varepsilon$ from (2.2) and the subsequent definitions without loss of crucial properties in general Banach spaces.

Given $\varphi: X \rightarrow \overline{\mathbb{R}}$ finite at $\bar{x}$, the (lower) basic subdifferential of $\varphi$ at $\bar{x}$ can be defined geometrically by

$$
\partial \varphi(\bar{x}):=\left\{x^{*} \in X^{*} \mid\left(x^{*},-1\right) \in N((\bar{x}, \varphi(\bar{x})) ; \operatorname{epi} \varphi)\right\}
$$

If $\varphi$ is lower semicontinuous (l.s.c.) around $\bar{x}$ and if $X$ is Asplund, construction (2.3) is equivalent to the analytic representation

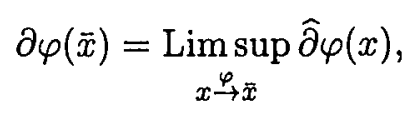


where $x \stackrel{\varphi}{\rightarrow} \bar{x}$ means that $x \rightarrow \bar{x}$ and $\varphi(x) \rightarrow \varphi(\bar{x})$. The basic upper superdifferential of $\varphi$ at $\bar{x}$ is defined by $\partial^{+} \varphi(\bar{x}):=-\partial(-\varphi)(\bar{x})$ and can be represented via basic normals to the hypograph of $\varphi$ as well as via sequential limits of Fréchet upper subgradients similarly to the basic subdifferential. Recall that $\varphi$ is lower regular (resp. upper regular) at $\bar{x}$ if

$$
\partial \varphi(\bar{x})=\widehat{\partial} \varphi(\bar{x}) \quad\left(\operatorname{resp} . \partial^{+} \varphi(\bar{x})=\widehat{\partial}^{+} \varphi(\bar{x})\right) .
$$

Upper regular functions are of special interest for this paper in connection with upper subdifferential optimality conditions. Note that this class contains, in particular, all concave continuous functions and all functions strictly differentiable at $\bar{x}$, as well as other functions $\varphi$ for which $-\varphi$ is lower regular at $\bar{x}$; cf. $[12,25]$. Note that $\widehat{\partial}^{+} \varphi(\bar{x}) \neq \emptyset$ if $\varphi$ is upper regular at $\bar{x}$ and Lipschitz continuous around this point while $X$ is Asplund. This follows from the fact that $\partial^{+} \varphi(\bar{x}) \neq \emptyset$ for any locally Lipschitzian function on an Asplund space; see [18, Corollary 3.9]. If $\varphi$ is concave and continuous around $\bar{x}$, then $\hat{\partial}^{+} \varphi(\bar{x}) \neq \emptyset$ in any Banach space, which is well known in convex analysis. Observe also that

$$
\bar{\partial} \varphi(\bar{x})=\mathrm{cl}^{*} \widehat{\partial}^{+} \varphi(\bar{x})
$$

for every function $\varphi$ on an Asplund space that is upper regular at $\bar{x}$ and Lipschitz continuous around this point, where $\bar{\partial} \varphi(\bar{x})$ stands for the Clarke generalized gradient [4], and where $\mathrm{cl}^{*}$ denotes the topological closure of a set in the weak* topology of $X^{*}$. Moreover, the weak* closure is redundant in (2.5) if $X$ is weakly compactly generated (WCG), in particular, it is either reflexive or separable. Indeed, by the symmetry property of the Clarke generalized gradient for locally Lipschitzian functions [4, Proposition 2.3.1], its representation through the basic subdifferential in Asplund spaces [18, Theorem 8.11], and the convexity of $\widehat{\partial}^{+} \varphi(\bar{x})$ one has

$$
\bar{\partial} \varphi(\bar{x})=-\bar{\partial}(-\varphi)(\bar{x})=-\operatorname{cl}^{*} \operatorname{co} \partial(-\varphi)(\bar{x})=\operatorname{cl}^{*} \operatorname{co} \partial^{+} \varphi(\bar{x})=\operatorname{cl}^{*} \hat{\partial}^{+} \varphi(\bar{x})
$$

where $\partial \varphi(\bar{x})$ and hence $\widehat{\partial}^{+} \varphi(\bar{x})$ are weak* closed in WCG spaces due to [18, Theorem 9.2].

Given a set-valued mapping $F: X \rightrightarrows Y$ between Banach spaces, we define its normal coderivaitive $D_{N}^{*} F(\bar{x}, \bar{y}): Y^{*} \rightarrow X^{*}$ at $(\bar{x}, \bar{y}) \in \operatorname{gph} F$ by

$$
D_{N}^{*} F(\bar{x}, \bar{y})\left(y^{*}\right):=\left\{x^{*} \in X^{*} \mid\left(x^{*},-y^{*}\right) \in N((\bar{x}, \bar{y}) ; \operatorname{gph} F)\right\}
$$

and the corresponding mixed coderivative by

$$
\begin{aligned}
D_{M}^{*} F(\bar{x}, \bar{y})\left(y^{*}\right):=\left\{x^{*} \in X^{*} \mid\right. & \exists \varepsilon_{k} \downarrow 0,\left(x_{k}, y_{k}\right) \rightarrow(\bar{x}, \bar{y}), x_{k}^{*} \stackrel{w^{*}}{\rightarrow} x^{*}, y_{k}^{*} \stackrel{\|\cdot\|}{\rightarrow} y^{*}, \\
& \text { with } \left.\left(x_{k}^{*},-y_{k}^{*}\right) \in \widehat{N}_{\varepsilon_{k}}\left(\left(x_{k}, y_{k}\right) ; \operatorname{gph} F\right), k \rightarrow \infty\right\},
\end{aligned}
$$

where $\varepsilon_{k}$ can be equivalently omitted if the graph of $F$ is closed around $(\bar{x}, \bar{y})$ and if both $X$ and $Y$ are Asplund. We also omit $\bar{y}$ in (2.6) and (2.7) when $F=f: X \rightarrow Y$ is single-valued and use the common coderivative symbol $D^{*} F$ if both coderivatives agree. This happens, in particular, when $Y$ is finite-dimensional, while the mixed coderivative may be strictly smaller (never bigger) than the normal coderivative even for single-valued Lipschitzian mappings into the Hilbert space $Y=\ell^{2}$. One has the scalarization formulas

$$
D_{M}^{*} f(\bar{x})\left(y^{*}\right)=\partial\left\langle y^{*}, f\right\rangle(\bar{x}), \quad D_{N}^{*} f(\bar{x})\left(y^{*}\right)=\partial\left\langle y^{*}, f\right\rangle(\bar{x})
$$


with $\left\langle y^{*}, f\right\rangle(x):=\left\langle y^{*}, f(x)\right\rangle$, where the first formula in (2.8) holds for every locally Lipschitzian mapping between Banach spaces, while the second scalarization formula is established in [18, Theorem 5.2] for the case of Asplund spaces $X$ and strictly Lipschitzian mappings $f$. The latter subclass of Lipschitzian mappings is proved to reduce to compactly Lipschitzian mappings in the sense of Thibault; see [26] for more details. If, in particular, $f$ is strictly differentiable at $\bar{x}$, then formulas (2.8) reduce both coderivatives to the adjoint derivative operator

$$
D_{M}^{*} f(\bar{x})\left(y^{*}\right)=D_{N}^{*} f(\bar{x})\left(y^{*}\right)=\left\{\nabla f(\bar{x})^{*} y^{*}\right\}, \quad y^{*} \in Y^{*}
$$

in any Banach spaces $X$ and $Y$. Using the coderivative $D^{*} E_{\varphi}(\ddot{x})$ of the epigraphical multifunction $E_{\varphi}(x):=\{\mu \in \mathbb{R} \mid \mu \geq \varphi(x)\}$ associated with $\varphi: X \rightarrow \overline{\mathbb{R}}$, we get back to the basic subdifferential $\partial \varphi(\bar{x})$ and define the (lower) singular subdifferential $\partial^{\infty} \varphi(\bar{x})$ of $\varphi$ at $\bar{x}$ by

$$
\partial \varphi(\bar{x})=D^{*} E_{\varphi}(\bar{x}, \varphi(\bar{x}))(1), \quad \partial^{\infty} \varphi(\bar{x}):=D^{*} E_{\varphi}(\bar{x}, \varphi(\bar{x}))(0)
$$

It is easy to see that $\partial^{\infty} \varphi(\bar{x})=\{0\}$ for locally Lipschitzian functions $\varphi$ on arbitrary Banach spaces.

In this paper we also use the construction of the (normal) second-order subdifferential of $\varphi: X \rightarrow$ $\overline{\mathbb{R}}$ at $\bar{x}$ relative to $\bar{y} \in \partial \varphi(\bar{x})$ defined by

$$
\partial_{N}^{2} \varphi(\bar{x}, \bar{y})(u):=D_{N}^{*}(\partial \varphi)(\bar{x}, \bar{y})(u), \quad u \in X^{* *} .
$$

The mixed second-order subdifferential is defined similarly, but we do not need it in what follows. Note that for $\varphi \in \mathcal{C}^{2}$ one has

$$
\partial_{N}^{2} \varphi(\bar{x})(u)=\left\{\nabla^{2} \varphi(x)^{*} u\right\}, \quad u \in X^{* *}
$$

where $\nabla^{2} \varphi(\bar{x})$ stands for the classical second-order derivative operator.

Next we recall certain normal compactness properties of sets from products of Banach spaces; see [19] and its references for the genesis of these and related properties and more discussions. A set $\Omega \subset X \times Y$ is sequentially normally compact (SNC) at $(\bar{x}, \bar{y}) \in \Omega$ if for any sequences $\left(\varepsilon_{k}, x_{k}, x_{k}^{*}, y_{k}^{*}\right) \in[0, \infty) \times \Omega \times X^{*} \times Y^{*}$ satisfying

$$
\varepsilon_{k} \downarrow 0, \quad\left(x_{k}, y_{k}\right) \rightarrow(\bar{x}, \bar{y}), \quad\left(x_{k}^{*}, y_{k}^{*}\right) \in \widehat{N}_{\varepsilon_{k}}\left(\left(x_{k}, y_{k}\right) ; \Omega\right)
$$

one has the implication

$$
\left(x_{k}^{*}, y_{k}^{*}\right) \stackrel{w^{*}}{\rightarrow}(0,0) \Longrightarrow\left\|\left(x_{k}^{*}, y_{k}^{*}\right)\right\| \rightarrow 0 \quad \text { as } \quad k \rightarrow \infty .
$$

This set is partially sequentially normally compact (PSNC) at $(\bar{x}, \bar{y})$ with respect to $X$ if for any above sequences satisfying (2.11) one has

$$
\left[x_{k}^{*} \stackrel{w^{*}}{\rightarrow} 0 \text { and }\left\|y_{k}^{*}\right\| \rightarrow 0\right] \Longrightarrow\left\|x_{k}^{*}\right\| \rightarrow 0 \text { as } k \rightarrow \infty
$$

Finally, $\Omega$ is strongly $P S N C$ at $(\bar{x}, \bar{y})$ with respect to $X$ if

$$
\left[\left(x_{k}^{*}, y_{k}^{*}\right) \stackrel{w^{*}}{\rightarrow}(0,0)\right] \Longrightarrow\left\|x_{k}^{*}\right\| \rightarrow 0 \text { as } k \rightarrow \infty .
$$

for every sequences satisfying (2.11). 
It follows from the definitions that

$$
\mathrm{SNC} \Longrightarrow \text { strong PSNC } \Longrightarrow \mathrm{PSNC}
$$

for any $(\bar{x}, \bar{y}) \in \Omega$ and that the above properties automatically hold in finite dimensions. Note that $\varepsilon_{k}$ may be equivalently omitted in (2.1) if both spaces $X, Y$ are Asplund and if $\Omega$ is locally closed around $(\bar{x}, \bar{y})$. Note also that the SNC property, in contrast to the other two, does not depend on the product structure on the Banach space in question. It is closely related to the compactly epi-Lipschitzian property of sets in the sense of [2], but the latter may be stronger in nonseparable Banach and Asplund spaces; see $[8,10]$ for recent comprehensive studies.

The corresponding SNC/PSNC properties of a set-valued mapping $F: X \rightarrow Y$ are defined via those for its graph at $(\bar{x}, \bar{y}) \in \operatorname{gph} F$. We omit "with respect to $X$ " when referring to the PSNC properties of mappings. Recall [13] that $F: X \Rightarrow Y$ is PSNC at $(\bar{x}, \bar{y})$, for any Banach spaces $X$ and $Y$, if it satisfies the Aubin Lipschitz-like property (known also as the "pseudo-Lipschitzian" property; see [1, 25]) around this point.

An extended-real-valued function $\varphi: X \rightarrow \bar{R}$ is sequentially normally epi-compact (SNEC) at $\bar{x}$ if its epigraph is SNC at $(\bar{x}, \varphi(\bar{x}))$. Note that if $\varphi: X \rightarrow \mathbb{R}$ is locally Lipschitzian around $\bar{x}$, it is $\mathrm{SNC}$ and hence SNEC at this point.

\section{Optimality Conditions under Geometric Constraints}

First let us derive necessary optimality conditions, of both lower and upper subdifferential types, for the initial problem (1.1) with the only (abstract) geometric constraint given by an arbitrary set $\Omega \subset X$ in infinite dimensions.

Theorem 3.1 (upper and lower subdifferential conditions for problems with abstract geometric constraints). Let $\bar{x}$ be a local optimal solution to the minimization problem (1.1) in a Banach space $X$ with $\left|\varphi_{0}(\bar{x})\right|<\infty$. Then the following hold:

(i) For every $x^{*} \in \widehat{\partial}^{+} \varphi_{0}(\bar{x})$ one has $-x^{*} \in \widehat{N}(\bar{x} ; \Omega)$, i.e.,

$$
-\widehat{\partial}^{+} \varphi_{0}(\bar{x}) \subset \widehat{N}(\bar{x} ; \Omega) \quad \text { and } \quad-\widehat{\partial}^{+} \varphi_{0}(\bar{x}) \subset N(\bar{x} ; \Omega) .
$$

(ii) Suppose that $X$ is Asplund, that $\varphi_{0}$ is l.s.c. around $\bar{x}$, and that $\Omega$ is locally closed around this point. Assume also that

$$
\partial^{\infty} \varphi_{0}(\bar{x}) \cap(-N(\bar{x} ; \Omega))=\{0\}
$$

and that either $\Omega$ is SNC at $\bar{x}$ or $\varphi_{0}$ is SNEC at $\bar{x}$; all these assumptions are satisfied if $\varphi_{0}$ is locally Lipschitzian around $\bar{x}$. Then one has

$$
\partial \varphi_{0}(\bar{x}) \cap(-N(\bar{x} ; \Omega)) \neq \emptyset, \quad \text { i.e. }, \quad 0 \in \partial \varphi_{0}(\bar{x})+N(\bar{x} ; \Omega) .
$$

Proof. Let us establish the upper subdifferential conditions in (i). Since $\widehat{N}(\bar{x} ; \Omega) \subset N(\bar{x} ; \Omega)$, we just need to prove the first inclusion in (3.1). Take any $x^{*} \in \widehat{\partial}^{+} \varphi_{0}(\vec{x})$ and observe that, in an arbitrary Banach space $X$, there is a function $s: X \rightarrow \mathbb{R}$ with

$$
s(\bar{x})=\varphi_{0}(\bar{x}) \text { and } s(x) \geq \varphi_{0}(x) \text { whenever } \quad x \in X
$$


such that $s(\cdot)$ is Fréchet differentiable at $\bar{x}$ with $\nabla s(\bar{x})=x^{*}$. Indeed, it follows directly from definition (1.6) that the function

$$
s(x):=\max \left\{\varphi_{0}(x), \varphi_{0}(\bar{x})+\left\langle x^{*}, x-\bar{x}\right\rangle\right\}
$$

enjoys all the above properties. One therefore has

$$
s(\bar{x})=\varphi_{0}(\bar{x}) \leq \varphi_{0}(x) \leq s(x) \text { for all } x \in \Omega,
$$

and thus $\bar{x}$ is a local optimal solution to the constrained minimization problem:

$$
\text { minimize } s(x) \text { subject to } x \in \Omega
$$

with a Fréchet differentiable objective. Applying now the necessary optimality condition (1.4) in the latter problem, we get

$$
-x^{*}=-\nabla s(\bar{x}) \in \hat{N}(\ddot{x} ; \Omega),
$$

which justifies the upper subdifferential optimality conditions (3.1) in general Banach spaces.

Next let us prove the subdifferential optimality condition (3.3) under the assumptions made in (ii). As mentioned in Section 1, one has inclusion (1.3) by the generalized Fermat rule. This immediately yields

$$
0 \in \partial\left(\varphi_{0}+\delta(\cdot ; \Omega)\right)(\bar{x})
$$

in terms of the basic subdifferential of the sum $\varphi_{0}+\delta(\cdot ; \Omega)$. Applying the subdifferential sum rule proved in [18, Theorem 4.1] to the latter sum and taking into account that

$$
\partial \delta(\bar{x} ; \Omega)=\partial^{\infty} \delta(\bar{x} ; \Omega)=N(\bar{x} ; \Omega)
$$

we arrive at (3.3) under the assumptions made. As mentioned above, $\varphi_{0}$ is SNEC at $\bar{x}$ and $\partial^{\infty} \varphi_{0}(\bar{x})=\{0\}$ (i.e., the qualification condition (3.2) automatically holds) if $\varphi_{0}$ is Lipschitz continuous around $\bar{x}$. This ends the proof of the theorem.

Note that the lower subdifferential optimality conditions in Theorem 3.1 apply to a very general class of extended-real-valued cost functions, while the upper subdifferential conditions in (i) is efficient only if $\hat{\partial}^{+} \varphi_{0}(\bar{x}) \neq \emptyset$. Nevertheless, the upper subdifferential conditions may give an essentially stronger result for special important classes of nonsmooth problems. In particular, for concave continuous functions $\varphi_{0}$ one has

$$
\partial \varphi_{0}(\bar{x}) \subset \partial^{+} \varphi_{0}(\bar{x})=\widehat{\partial}^{+} \varphi_{0}(\bar{x}) \neq \emptyset
$$

Then comparing the second inclusion in (3.1) (which is even weaker than the first inclusion therein) with the one in (3.3), we see that the upper subdifferential necessary condition requires that every element $x^{*}$ of the set $\widehat{\partial}^{+} \varphi_{0}(\bar{x})$ must belong to $-N(\bar{x} ; \Omega)$, instead of that some element $x^{*}$ from the smaller set $\partial \varphi_{0}(\bar{x})$ belongs to $-N(\bar{x} ; \Omega)$ by the lower subdifferential one. This shows that the upper subdifferential necessary conditions for local minima may have sizeable advantages over the 
lower subdifferential conditions above when the former efficiently apply. For example, consider the following simple one-dimensional problem:

$$
\text { minimize } \varphi_{0}(x):=-|x| \text { subject to } x \in \Omega:=[-1,0] \subset \mathbb{R} .
$$

Obviously $\bar{x}=0$ is not an optimal solution to this problem. However, it cannot be eliminated by the lower subdifferential condition (3.3), which is satisfied:

$$
\partial \varphi(0)=\{-1,1\}, \quad N(0 ; \Omega)=[0, \infty), \quad \text { and } \quad-1 \in-N(0, \Omega) .
$$

On the other hand, the upper subdifferential conditions in (3.1), which are the same in this case, do not hold for $\bar{x}=0$ giving

$$
\widehat{\partial}^{+} \varphi(0)=[-1,1] \text { and }[-1,1] \not \subset N(0 ; \Omega) .
$$

Recall also that $\widehat{\partial}^{+} \varphi_{0}(\bar{x}) \neq \emptyset$ if $\varphi_{0}$ is locally Lipschitzian and upper regular at $\bar{x}$ while $X$ is Asplund. Moreover, $\bar{\partial} \varphi_{0}(\bar{x})=\widehat{\partial}^{+} \varphi_{0}(\bar{x})$ for the Clarke generalized gradient if in addition $X$ is WCG; see (2.5). Thus in this case we have

$$
-\bar{\partial} \varphi_{0}(\bar{x}) \subset \widehat{N}(\bar{x} ; \Omega) \subset N(\bar{x} ; \Omega)
$$

by (3.1) instead of $\bar{\partial} \varphi_{0}(\bar{x}) \cap\left(-\mathrm{cl}^{*} \operatorname{co} N(\bar{x} ; \Omega)\right) \neq \emptyset$ by Clarke's counterpart; cf. [4].

Now let us consider minimization problems with finitely many geometric constraints that typically arise in applications. Having in mind particular applications in Sections 4 and 5 of this paper, we pay the main attention to problems with geometric constraints given by two set intersections:

$$
\text { minimize } \varphi_{0}(z) \text { subject to } z \in \Omega_{1} \cap \Omega_{2} \text {. }
$$

Most results for problems with finitely many geometric constraints can be reduced to the case of two constraint problems (3.4) by induction.

To derive more general and powerful results needed for subsequent applications, we consider problems (3.4) given in spaces with a product structure $X \times Y$ that particularly occurs in the framework of mathematical programs with equilibrium constraints; see Section 5 . The next theorem gives both upper and lower subdifferential optimality conditions for such problems.

Theorem 3.2 (upper and lower subdifferential conditions for problems with many geometric constraints). Let $\bar{z}$ be a local optimal solution to problem (3.4), where the sets $\Omega_{1}, \Omega_{2} \subset X \times Y$ are locally closed around $\bar{z}$, and where both spaces $X$ and $Y$ are Asplund. The following hold:

(i) Assume that the set system $\left\{\Omega_{1}, \Omega_{2}\right\}$ satisfies the limiting qualification condition at $\bar{z}$ : for any sequences $z_{i k} \stackrel{\Omega_{i}}{\rightarrow} \bar{z}$ and $z_{i k}^{*} \stackrel{w^{*}}{\rightarrow} z_{i}^{*}$ as $k \rightarrow \infty$ with $z_{i k}^{*} \in \widehat{N}\left(z_{i k} ; \Omega_{i}\right), i=1,2$, one has

$$
\left\|z_{1 k}^{*}+z_{2 k}^{*}\right\| \rightarrow 0 \Longrightarrow z_{1}^{*}=z_{2}^{*}=0 .
$$

Suppose also that either one of the sets $\Omega_{i}$ is $S N C$ at $\bar{z}$, or $\Omega_{1}$ is PSNC at $\vec{z}$ with respect to $X$ while $\Omega_{2}$ is strongly PSNC at this point with respect to $Y$. Then

$$
-\hat{\partial}^{+} \varphi_{0}(\bar{z}) \subset N\left(\bar{z} ; \Omega_{1}\right)+N\left(\bar{z} ; \Omega_{2}\right) .
$$


(ii) In addition to the assumptions in (i), suppose that $\varphi_{0}$ is l.s.c. around $\bar{z}$ and $S N E C$ at this point and that

$$
\left(-\partial^{\infty} \varphi_{0}(\bar{z})\right) \cap\left[N\left(\bar{z} ; \Omega_{1}\right)+N\left(\bar{z} ; \Omega_{2}\right)\right]=\{0\}
$$

(all the additional assumptions are satisfied if $\varphi_{0}$ is Lipschitz continuous around $\bar{z}$ ). Then one has

$$
0 \in \partial \varphi_{0}(\bar{z})+N\left(\bar{z} ; \Omega_{1}\right)+N\left(\bar{z} ; \Omega_{2}\right) .
$$

(iii) Assume that $\varphi_{0}$ is l.s.c. around $\bar{z}$, that both $\Omega_{1}$ and $\Omega_{2}$ are $S N C$ at this point, and that the qualification condition

$$
\begin{aligned}
& {\left[z_{0}^{*} \in \partial^{\infty} \varphi_{0}(\bar{z}), \quad z_{1}^{*} \in N\left(\bar{z} ; \Omega_{1}\right), \quad z_{2}^{*} \in N\left(\bar{z} ; \Omega_{2}\right),\right.} \\
& \left.z_{0}^{*}+z_{1}^{*}+z_{2}^{*}=0\right] \Longrightarrow z_{0}^{*}=z_{1}^{*}=z_{2}^{*}=0
\end{aligned}
$$

holds. Then one has (3.8).

Proof. To prove (i), we base on the second upper subdifferential inclusion in Theorem 3.1(i) involving the basic normal cone to $\Omega:=\Omega_{1} \cap \Omega_{2}$. This gives

$$
-\hat{\partial}^{+} \varphi_{0}(\bar{z}) \subset N\left(\bar{z} ; \Omega_{1} \cap \Omega_{2}\right) \text {. }
$$

Now we can use the intersection rule for the basic normal cone to $\Omega_{1} \cap \Omega_{2}$ that is not available for Fréchet normals in (3.1). Employing the general result of [19, Theorem 4.2], one has

$$
N\left(\bar{z} ; \Omega_{1} \cap \Omega_{2}\right) \subset N\left(\bar{z} ; \Omega_{1}\right)+N\left(\bar{z} ; \Omega_{2}\right)
$$

under the limiting qualification condition and the SNC/PSNC assumptions made in (i). Thus we arrive at the upper subdifferential inclusion (3.6).

Assertion (ii) of the theorem follows from Theorem 3.1(ii) under the SNEC assumption on $\varphi_{0}$ and from the above intersection rule of [19, Theorem 4.2] by substituting (3.10) into (3.2) and (3.3).

It remains to prove (iii). Using Theorem 3.1(ii) in the case of SNC sets $\Omega$, we need to express the SNC assumption on $\Omega$ and the other conditions of that theorem in terms of $\Omega_{1}, \Omega_{2}$, and $\varphi_{0}$. To proceed, one needs to employ the SNC preservation/calculus rules developed in [20]. In particular, corollary 3.6 of that paper ensures the SNC property of the intersection $\Omega_{1} \cap \Omega_{2}$ at $\bar{z}$ provided that both $\Omega_{i}$ are SNC at this point and that the qualification condition

$$
N\left(\bar{z} ; \Omega_{1}\right) \cap\left(-N\left(\bar{z} ; \Omega_{2}\right)\right)=\{0\}
$$

is satisfied. These assumptions automatically guarantee the fulfillment of the intersection rule (3.10). It is easy to check that (3.9) implies both qualification conditions (3.2) at $\bar{z}$ and (3.11). Indeed, (3.11) follows right from (3.9) with $z_{0}^{*}=0$. To get (3.2) at $\bar{z}$, we take $z_{0}^{*} \in N\left(\bar{z} ; \Omega_{1} \cap \Omega_{2}\right)$ with $-z_{0}^{*} \in \partial^{\infty} \varphi_{0}(\bar{z})$ and find $z_{i}^{*} \in N\left(\bar{z} ; \Omega_{i}\right), i=1,2$, such that $z_{1}^{*}+z_{2}^{*}=z_{0}^{*}$ by (3.10). Thus $z_{0}^{*}+z_{1}^{*}+z_{2}^{*}=0$, which gives $z_{0}^{*}=0$ by $(3.9)$ and ends the proof of the theorem.

As observed, the normal qualification condition (3.11) implies the limiting one in Theorem 3.2. Indeed, the former corresponds to the replacement of the implication in (3.5) by

$$
z_{1 k}^{*}+z_{2 k}^{*} \stackrel{w^{*}}{\rightarrow} 0 \Longrightarrow z_{1}^{*}=z_{2}^{*}=0 .
$$

We will see in Section 5 that, being applied to graphs of set-valued mappings, the limiting qualification condition of Theorem 3.2 has essential advantages in comparison with the normal qualification condition (3.11). 


\section{Optimality Conditions under Operator and Functional Con- straints}

In this section we derive necessary optimality conditions of both lower and upper subdifferential types for minimization problems that contain, along with geometric constraints, also constraints given by set-valued and single-valued mappings/operators between possibly infinite-dimensional spaces, as well as in more conventional forms involving real-valued functions. The general problem under consideration is as follows:

$$
\text { minimize } \varphi_{0}(x) \text { subject to } x \in F^{-1}(\Theta) \cap \Omega \text {, }
$$

where $\varphi_{0}: X \rightarrow \overline{\mathbb{R}}, F: X \Rightarrow Y, \Omega \subset X, \Theta \subset Y$, and where

$$
F^{-1}(\Theta):=\{x \in X \mid F(x) \cap \Theta \neq \emptyset\}
$$

in the inverse image of the set $\Theta$ under the set-valued mapping $F$ between Banach spaces. Model (4.1) covers many special classes of optimization problems, in particular, classical problems of nonlinear programming with equality and inequality constraints.

Observe that (4.1) reduces to the problem of constrained minimization admitting only geometric constraints given by the intersection of two sets: $\Omega_{1}=F^{-1}(\Theta)$ and $\Omega_{2}=\Omega$. Thus one can apply the results of the preceding subsection and then calculus rules for basic normals to inverse images and intersections as well as those preserving SNC properties. In this way we arrive at necessary optimality conditions of the lower and upper subdifferential types in both normal (Kuhn-Tucker) form under some constraint qualifications ensuring a nonzero multiplier associated with the cost function, as well as a non-qualified (Fritz John) form that does not impose constraint qualifications and does not ensure the nontriviality of the above multiplier. For brevity we present here only some results in the latter form.

Let us start with upper subdifferential conditions. Recall that a set-valued mapping $M: X \rightrightarrows Y$ is inner semicompact at $\bar{x}$ with $M(\bar{x}) \neq \emptyset$ if for every sequence $x_{k} \rightarrow \bar{x}$ with $M\left(x_{k}\right) \neq \emptyset$ there is a sequence $y_{k} \in M\left(x_{k}\right)$ that contains a convergent subsequence. We say that $M(\cdot)$ is inner semicompact around $\bar{x}$ if this property holds for every $x$ in some neighborhood of $\bar{x}$. The latter property obviously holds for set-valued mappings that are locally compact (locally bounded when $\operatorname{dim} Y<\infty)$ around the reference point.

Theorem 4.1 (upper subdifferential optimality conditions under operator constraints). Given a local optimal solution $\bar{x}$ to problem (4.1), we have the following assertions:

(i) Assume that $X$ and $Y$ are Banach, that $\Omega=X$ and $\Theta=\{0\}$, and that $F=f: X \rightarrow Y$ is Fréchet differentiable at $\bar{x}$. Then there exists $\lambda_{0} \geq 0$ such that for every $x_{0}^{*} \in \hat{\partial}^{+} \varphi_{0}(\bar{x})$ there is $y^{*} \in Y^{*}$ for which

$$
0=\lambda_{0} x_{0}^{*}+\nabla f(\bar{x})^{*} y^{*}, \quad\left(\lambda_{0}, y^{*}\right) \neq 0,
$$

provided that either $f$ is strictly differentiable at $\bar{x}$ or $\operatorname{dim} Y<\infty$.

(ii) Assume that $X$ is Asplund while $Y$ is Banach, that $f: X \rightarrow Y$ is strictly differentiable at $\bar{x}$ with the surjective derivative $\nabla f(\bar{x})$, and that $\Omega$ is locally closed around $\bar{x}$. Then there exists $\lambda_{0} \geq 0$ such that for every $x_{0}^{*} \in \widehat{\partial}^{+} \varphi_{0}(\bar{x})$ there is $y^{*} \in N(f(\bar{x}) ; \Theta)$ for which

$$
-\lambda_{0} x_{0}^{*}-\nabla f(\bar{x})^{*} y^{*} \in N(\bar{x} ; \Omega), \quad\left(\lambda_{0}, y^{*}\right) \neq 0
$$


provided that either $\Omega$ is $S N C$ at $\bar{x}$ or $\Theta$ is $S N C$ at $f(\bar{x})$.

(iii) Assume that both $X$ and $Y$ are Asplund, that $\Omega$ and $\Theta$ are closed, and that $M(\cdot):=F(\cdot) \cap \Theta$ is inner semicompact around $\bar{x}$. Then there exists $\lambda_{0} \geq 0$ such that for every $x_{0}^{*} \in \widehat{\partial}^{+} \varphi_{0}(\bar{x})$ there are $\bar{y} \in M(\bar{x})$ and dual elements $y^{*} \in N(\bar{y} ; \Theta), x_{1}^{*} \in D_{N}^{*} F(\bar{x}, \bar{y})\left(y^{*}\right)$, and $x_{2}^{*} \in N(\bar{x} ; \Omega)$ satisfying

$$
0=\lambda_{0} x_{0}^{*}+x_{1}^{*}+x_{2}^{*}, \quad\left(\lambda_{0}, y^{*}, x_{1}^{*}\right) \neq 0,
$$

provided that one of the following properties holds for every $\bar{y} \in M(\bar{x})$ :

(a) $\Omega$ is $S N C$ at $\bar{x}$ and $F^{-1}$ is PSNC at $(\bar{y}, \bar{x})$;

(b) $\Omega$ is $S N C$ at $\bar{x}$ and $\Theta$ is $S N C$ at $\bar{y}$;

(c) $F$ is PSNC at $(\bar{x}, \bar{y})$ and $\Theta$ is $S N C$ at $\bar{y}$;

(d) $F$ is $S N C$ at $(\bar{x}, \bar{y})$.

Proof. To prove (i) in the general Banach space setting, we first assume that $f$ is Fréchet differentiable at $\vec{x}$ with the surjective derivative $\nabla f(\bar{x})$. Then for any set $\Theta \subset Y$ with $f(\bar{x}) \in \Theta$ one has

$$
\widehat{N}\left(\bar{x} ; f^{-1}(\Theta)\right)=\nabla f(\bar{x})^{*} \widehat{N}(f(\bar{x}) ; \Theta),
$$

which follows from [21, Theorem 3.1] and the Lyusternik-Graves theorem on metric regularity. Since the proof in [21] requires the metric regularity just at (but not around) the reference point, it ensures the fulfillment of (4.5) also in the case when $f$ is merely Fréchet differentiable at $\vec{x}$ with the surjective derivative provided that $Y$ is finite-dimensional. It can be done by using the Brouwer fixed point theorem instead of the Lyusternik-Graves result; $c f$. the arguments in [9, Proposition 7 ] establishing a somewhat different but related controllability property. Then substituting (4.5) into the first inclusion in (3.1) with $\Omega=f^{-1}(\Theta)$, we get

$$
-\widehat{\partial}^{+} \varphi_{0}(\bar{x}) \subset \nabla f(\bar{x})^{*} \widehat{N}(f(\bar{x}) ; \Theta) .
$$

For $\Theta=\{0\}$ the latter gives (4.2) with $\lambda_{0}=1$ under the surjectivity assumption on $\nabla f(\bar{x})$. If $\nabla f(\bar{x})$ is not surjective, then $\operatorname{ker} \nabla f(\bar{x})^{*} \neq\{0\}$, i.e., there is $0 \neq y^{*} \in Y^{*}$ such that $\nabla f(\bar{x})^{*} y^{*}=0$. Thus we get (4.2) with $\lambda_{0}=0$ and $y^{*} \neq 0$.

To prove (ii) when $X$ is Asplund (while $Y$ may be arbitrarily Banach) and $f$ is strictly differentiable at $\bar{x}$ with the surjective derivative, we apply assertion (i) of Theorem 3.2 with $\Omega_{1}=f^{-1}(\Theta)$ and $\Omega_{2}=\Omega$ assuming that either $\Omega$ or $f^{-1}(\Theta)$ is SNC at $\bar{x}$ and $f(\bar{x})$, respectively, and that

$$
N\left(\bar{x} ; f^{-1}(\Theta)\right) \cap(-N(\bar{x} ; \Omega))=\{0\} .
$$

When $\Omega$ is SNC at $\bar{x}$, this yields

$$
-\widehat{\partial}^{+} \varphi_{0}(\bar{x}) \subset \nabla f(\bar{x})^{*} N(f(\bar{x}) ; \Theta)+N(\bar{x} ; \Omega)
$$

under the qualification condition

$$
\nabla f(\bar{x})^{*} N(f(\bar{x}) ; \Theta) \cap(-N(\bar{x} ; \Omega))=\{0\} .
$$

Indeed, it follows from the the basic normal cone counterpart of equality (4.5) established in [21, Corollary 3.9] for any mapping $f$ between Banach spaces that is strictly differentiable at $\bar{x}$ with the 
surjective derivative. The latter assumptions ensure by [21, Corollary 5.3] that the SNC property of $f^{-1}(\Theta)$ at $\bar{x}$ is equivalent to the one for $\Theta$ at $f(\vec{x})$. Thus (4.6) implies (4.3) with $\lambda_{0}=1$ under the qualification condition (4.7) and the assumptions made in (ii). The negation of (4.7) means that (4.3) holds with $\lambda_{0}=0$ and $y^{*} \neq 0$, which completes the proof of (ii).

It remains to prove (iii). Again applying the upper subdifferential assertion (i) of Theorem 3.2 with $\Omega_{1}=F^{-1}(\Theta)$ and $\Omega_{2}=\Omega$, we now are able to proceed with a general case of set-valued mappings $F$ in the operator constraints of (4.1) having in hands the powerful tools of comprehensive calculus rules (including those for the preservation of SNC properties) in the Asplund space settings. First observe that the set $F^{-1}(\Theta)$ is locally closed around $\vec{x}$ due to the closedness and inner semicompactness assumptions made in (iii). Hence, by Theorem 3.2(i), one has

$$
-\widehat{\partial}^{+} \varphi_{0}(\bar{x}) \subset N\left(\bar{x} ; F^{-1}(\Theta)\right)+N(\bar{x} ; \Omega)
$$

provided the qualification condition

$$
N\left(\bar{x} ; F^{-1}(\Theta)\right) \cap(-N(\bar{x} ; \Omega))=\{0\}
$$

and that either $\Omega$ or $F^{-1}(\Theta)$ is SNC at $\bar{x}$. The SNC calculus result of [20, Theorem 3.8] ensures the latter property of the inverse image $F^{-1}(\Theta)$ under the qualification condition

$$
N(\bar{y} ; \Theta) \cap \operatorname{ker} D_{N}^{*} F(\bar{x}, \bar{y})=\{0\} \text { for all } \bar{y} \in M(\bar{x})
$$

assuming also that either $F$ is PSNC at $(\bar{x}, \bar{y})$ and $\Theta$ is SNC at $\bar{y}$, or $F$ is SNC at $(\bar{x}, \bar{y})$ for every $\bar{y} \in M(\bar{x})$. Now we apply the calculus rule of [19, Theorem 4.4] providing the inclusion

$$
N\left(\bar{x} ; F^{-1}(\Theta)\right) \subset \bigcup\left[D_{N}^{*} F(\bar{x}, \bar{y})\left(y^{*}\right) \mid \bar{y} \in M(\bar{x}), y^{*} \in N(\bar{y} ; \Theta)\right]
$$

under the qualification condition (4.10) and the assumptions that either $F^{-1}$ is PSNC at $(\bar{y}, \bar{x})$ or $\Theta$ is SNC at $\bar{y}$ for all $\bar{y} \in M(\bar{x})$. Substituting (4.11) into (4.8) and (4.9) and combining the SNC/PSNC assumptions made on $\Omega, \Theta, F$, and $F^{-1}$ above, we arrive at the upper subdifferential optimality condition

$$
-\widehat{\partial}^{+} \varphi_{0}(\bar{x}) \subset \bigcup\left[D_{N}^{*} F(\bar{x}, \bar{y})\left(y^{*}\right) \mid \bar{y} \in M(\bar{x}), y^{*} \in N(\bar{y} ; \Theta)\right]+N(\bar{x} ; \Omega)
$$

under one of the assumptions (a)-(d) in (iii) and the constraint qualifications (4.10) and

$$
\bigcup\left[D_{N}^{*} F(\bar{x}, \bar{y})\left(y^{*}\right) \mid \bar{y} \in M(\bar{x}), y^{*} \in N(\bar{y} ; \Theta)\right] \cap(-N(\bar{x} ; \Omega))=\{0\}
$$

which therefore ensure the result of (iii) in the normal form $\left(\lambda_{0}=1\right)$.

If the above constraint qualifications are not satisfied, we have the optimality conditions in (iii) of the Fritz John type, i.e., with $\lambda_{0}$ in (4.2) possibly equal to zero but then either $y^{*}$ or $x_{1}^{*}$ is not. Indeed, when (4.10) is not satisfied, there are $\bar{y} \in M(\bar{x})$ and $0 \neq y^{*} \in N(\bar{y} ; \Theta)$ such that $0 \in D_{N}^{*} F(\bar{x}, \bar{y})\left(y^{*}\right)$. This gives (4.2) with $\lambda_{0}=0, y^{*} \neq 0, x_{1}^{*}=x_{2}^{*}=0$. If (4.13) is not satisfied, then there are $\bar{y} \in M(\bar{x})$ and $y^{*} \in N(\bar{y} ; \Theta), 0 \neq x^{*} \in D_{N}^{*} F(\bar{x}, \bar{y})\left(y^{*}\right)$ such that $-x^{*} \in N(\bar{x} ; \Omega)$. This gives (4.2) with $\lambda_{0}=0, x_{1}^{*}=-x_{2}^{*}=x^{*}$, which completes the proof of the theorem.

Next let us derive lower subdifferential conditions for problems (4.1) with general constraints. To furnish this, we are based on the subdifferential conditions of Theorem 3.2(ii) and the calculus 
rules as in the proof of the previous theorem assuming for simplicity that $\varphi_{0}$ is Lipschitz continuous around the reference point. In this way one may also derive lower subdifferential conditions in (4.1) for problems with non-Lipschitzian cost functions based on the corresponding results of Theorem 3.2. For brevity we only present below a lower subdifferential counterpart of assertion (iii) in Theorem 4.1.

Theorem 4.2 (upper subdifferential optimality conditions under operator constraints). Let $\bar{x}$ be a local optimal solution to problem (4.1). In addition to the assumptions of Theorem 4.1(iii), suppose that $\varphi_{0}$ is Lipschitz continuous around $\bar{x}$. Then there are $\lambda_{0} \geq 0, x_{0}^{*} \in \partial \varphi_{0}(\bar{x}), \bar{y} \in M(\bar{x})$, $y^{*} \in N(\bar{y} ; \Theta), x_{1}^{*} \in D_{N}^{*} F(\bar{x}, \bar{y})\left(y^{*}\right)$, and $x_{2}^{*} \in N(\bar{x} ; \Omega)$ such that $(4.4)$ holds provided that one of the properties (a)-(d) in (iii) of Theorem 4.1 is fulfilled for every $\bar{y} \in M(\bar{x})$.

Proof. The proof is based on the lower subdifferential inclusion

$$
0 \in \partial \varphi_{0}(\bar{x})+N\left(\bar{z} ; F^{-1}(\Theta)\right)+N(\bar{x} ; \Omega)
$$

from Theorem 3.2(ii) and the usage of calculus rules as in the proof of Theorem 4.1(iii).

Both upper and lower subdifferential conditions obtained and Theorems 4.1 and 4.2 allow significant simplifications when the operator constraints in (4.1) are given by single-valued and strictly Lipschitzian mappings.

Corollary 4.3 (optimality conditions under strictly Lipschitzian constraints). Let $\bar{x}$ be a local optimal solution to problem (4.1), where $X$ and $Y$ are Asplund, $\Omega$ and $\Theta$ are closed, and $F=f: X \rightarrow Y$ is single-valued and strictly Lipschitzian around $\bar{x}$. Then there exists $\lambda_{0} \geq 0$ such that for every $x^{*} \in \hat{\partial}^{+} \varphi_{0}(\bar{x})$ there is $y^{*} \in N(f(\bar{x}) ; \Theta)$ satisfying

$$
-\lambda_{0} x^{*} \in \partial\left\langle y^{*}, f\right\rangle(\bar{x})+N(\bar{x} ; \Omega), \quad\left(\lambda_{0}, y^{*}\right) \neq 0,
$$

provided that one of the following properties is fulfilled:

(a) $\Omega$ is SNC at $\bar{x}$ and $f^{-1}$ is PSNC at $(f(\bar{x}), \bar{x})$;

(b) $\Theta$ is $S N C$ at $f(\bar{x})$.

If in addition $\varphi_{0}$ is Lipschitz continuous around $\bar{x}$, then there are $\lambda_{0} \geq 0$ and $y^{*} \in N(f(\bar{x}) ; \Theta)$ satisfying

$$
0 \in \lambda_{0} \partial \varphi_{0}(\bar{x})+\partial\left\langle y^{*}, f\right\rangle(\bar{x})+N(\bar{x} ; \Omega), \quad\left(\lambda_{0}, y^{*}\right) \neq 0
$$

provided that either (a) or (b) holds.

Proof. These results follow from Theorems 4.1(iii) and 4.2, respectively, due to the normal scalarization formula (2.8), which ensures that $x_{1}^{*}=0$ if $y^{*}=0$ in the conditions above. In this case the requirements in (b) and (c) of Theorem 4.1 reduce to the SNC property of $\Theta$ at $f(\bar{x})$, since $f$ is automatically PSNC $\bar{x}$ due to its locally Lipschitz continuity. Let us mention that the SNC property of $f$ in (d) of Theorem 4.1 is redundant for the case of strictly Lipschitzian mappings. Indeed, one can show by using the classical Josefson-Nissenzweig theorem on the weak* convergence to zero of some sequence of unit dual vectors in every infinite-dimensional Banach space (see, e.g., the proof 
of [21, Theorem 5.1]) that a strictly Lipschitzian mapping $f: X \rightarrow Y$ is SNC at $\bar{x}$ if and only if $Y$ is finite-dimensional. Thus properties (a)-(d) in Theorem 4.1 reduce to (a) and (b) in the corollary.

The lower subdifferential optimality conditions of Theorem 4.2 and Corollary 4.3 improve and extend previous results obtained for minimization problems in terms of basic normals and subgradients under consideration in finite-dimensional and Asplund space settings; see, in particular, the results and comments in $[3,12,14,25]$ and the references therein. The upper subdifferential results obtained above seem to be new in the optimization theory.

Let us consider a special class of problems (4.1) concerning nondifferentiable programming with finitely many functional constraints if equality and inequality types given by

$$
\left\{\begin{array}{l}
\text { minimize } \varphi_{0}(x) \text { subject to } x \in \Omega, \\
\varphi_{i}(x) \leq 0, \quad i=1, \ldots, m, \\
\varphi_{i}(x)=0, \quad i=m+1, \ldots, m+r
\end{array}\right.
$$

where $\varphi_{i}: X \rightarrow \mathbb{R}$ for $i=0, \ldots, m+r$ and $\Omega \subset X$. The latter problem corresponds to (4.1) with the single-valued mapping $F=\left(\varphi_{1}, \ldots, \varphi_{m+r}\right): X \rightarrow \mathbb{R}^{m+r}$ and the closed convex cone

$$
\Theta:=\left\{\left(\alpha_{1}, \ldots, \alpha_{m+r}\right) \in \mathbb{R}^{m+r} \mid \begin{array}{l}
\alpha_{i} \leq 0 \text { for } i=1, \ldots, m \text { and } \\
\left.\alpha_{i}=0 \text { for } i=m+1, \ldots, m+r\right\} .
\end{array}\right.
$$

Taking into account relationships (2.8) and (2.9) between the coderivatives and subdifferentials, one can easily deduce from Theorems 4.1, 4.2 and Corollary 4.3 necessary optimality conditions for (4.14) involving (lower) basic and singular subgradients of the constraint functions; cf. [14, Theorem 5.1].

Now we present new necessary optimality conditions of the upper subdifferential type specific for problems (4.14), which involve Fréchet upper subgradients not only of cost functions but also of those describing inequality constraints. To proceed, we use variational descriptions of Fréchet subgradients in a subclass of Asplund spaces admitting Lipschitzian $\mathcal{C}^{1}$ bump functions, which is automatic in Banach spaces with Fréchet differentiable renorms, in particular, in any reflexive space; see [7] and its references.

Theorem 4.4 (upper subdifferential conditions in nondifferentiable programming). Let $\bar{x}$ be a local optimal solution to problem (4.14), where the set $\Omega$ is locally closed around $\bar{x}$ and the functions $\varphi_{i}$ are continuous around this point for $i=m+1, \ldots, m+r$. Suppose also that $X$ admits a Lipschitzian $\mathcal{C}^{1}$ bump function and that either $\Omega$ or $f:=\left(\varphi_{m+1}, \ldots, \varphi_{m+r}\right)$ is SNC at $\vec{x}$. Then for any Fréchet supergradients $x_{i}^{*} \in \widehat{\partial}^{+} \varphi_{i}(\bar{x}), i=0, \ldots, m$, there are $\left(\lambda_{0}, \ldots, \lambda_{m+r}\right) \in \mathbb{R}^{m+r+1}$, $x^{*} \in D^{*} f(\bar{x})\left(\lambda_{m+1}, \ldots, \lambda_{m+r}\right)$, and $\widetilde{x}^{*} \in N(\bar{x} ; \Omega)$ satisfying the relations

$$
\begin{gathered}
\lambda_{i} \geq 0 \text { for } i=0, \ldots, m, \quad \lambda_{i} \varphi_{i}(\bar{x})=0 \text { for } i=1, \ldots, m, \\
0=\sum_{i=0}^{m} \lambda_{i} x_{i}^{*}+x^{*}+\tilde{x}^{*}, \quad\left(\lambda_{0}, \ldots, \lambda_{m+r}, x^{*}\right) \neq 0 .
\end{gathered}
$$

If $\varphi_{i}$ are Lipschitz continuous around $\vec{x}$ for $i=m+1, \ldots, m+r$, then in addition to (4.16) one has

$$
-\sum_{i=0}^{m} \lambda_{i} x_{i}^{*} \in \partial\left(\sum_{i=m+1}^{m+r} \lambda_{i} \varphi_{i}\right)(\bar{x})+N(\bar{x} ; \Omega), \quad\left(\lambda_{0}, \ldots, \lambda_{m+r}\right) \neq 0
$$


with no other assumptions on $\left(\varphi_{i}, \Omega\right)$ besides the local closedness of $\Omega$.

Proof. Take arbitrary $x_{i}^{*} \in \widehat{\partial}^{+} \varphi_{i}(\bar{x})$ for $i=0, \ldots, m$ and apply the variational description from [7, Theorem 4.6(ii)] with $\mathcal{S}=\mathcal{L C}^{1}$ therein to the Fréchet subgradients $-x_{i}^{*} \in \hat{\partial}\left(-\varphi_{i}\right)(\bar{x})$. In this way we find functions $s_{i}: X \rightarrow \mathbb{R}$ for $i=0, \ldots, m$ satisfying $s_{i}(\bar{x})=\varphi_{i}(\bar{x}), s_{i}(x) \geq \varphi_{i}(x)$ for all $x$ from some neighborhood of $\bar{x}$, and such that each $s_{i}(x)$ is continuously differentiable around $\bar{x}$ with $\nabla s_{i}(\bar{x})=x_{i}^{*}$. It is easy to check that $\bar{x}$ is a local solution to the following optimization problem of type (4.14), where the cost and inequality constraint functions are continuously differentiable around this point:

$$
\left\{\begin{array}{l}
\operatorname{minimize} s_{0}(x) \quad \text { subject to } x \in \Omega \\
s_{i}(x) \leq 0, \quad i=1, \ldots, m, \\
\varphi_{i}(x)=0, \quad i=m+1, \ldots, m+r
\end{array}\right.
$$

Apply now the necessary conditions of Theorem 4.1(iii) to problem (4.19), which corresponds to (4.1) with the single-valued mapping $F:=\left(s_{1}, \ldots, s_{m}, \varphi_{m+1}, \ldots, \varphi_{m+r}\right)$ and the set $\Theta$ defined in (4.15). Observe that

$$
N\left(\left(\varphi_{1}(\bar{x}), \ldots, \varphi_{m+r}(\bar{x})\right) ; \Theta\right)=\left\{\begin{array}{l}
\left(\lambda_{1}, \ldots, \lambda_{m+r}\right) \in \mathbb{R}^{m+r} \mid \lambda_{i} \geq 0 \\
\left.\lambda_{i} \varphi_{i}(\bar{x})=0 \text { for } i=1, \ldots, m\right\}
\end{array}\right.
$$

with $s_{i}(\bar{x})=\varphi_{i}(\bar{x}), i=1, \ldots, m$, and that

$$
F(x)=(s(x), 0)+\left(0, \varphi_{m+1}(x), \ldots, \varphi_{m+r}(x)\right)
$$

for the above $F$, where $s:=\left(s_{1}, \ldots, s_{m}\right): X \rightarrow \mathbb{R}^{m}$ is continuously differentiable around $\bar{x}$. Thus the condition $y^{*} \in N(\dot{y} ; \Theta)$ in Theorem 4.1(iii) with $y^{*}=\left(\lambda_{1}, \ldots, \lambda_{m+r}\right)$ reduces to the sign and complementary slackness conditions in (4.16) as $i=1, \ldots, m$. Since $Y=\mathbb{R}^{m+r}$ in Theorem 4.1(iii), one can directly check that the SNC and PSNC properties of $F$ in (4.20) are equivalent to the SNC property of $f=\left(\varphi_{m+1}, \ldots, \varphi_{m+r}\right)$. It is easy also to see that one of the requirements (a)-(d) in Theorem 4.1(iii) holds if and only if either $\Omega$ or $f$ is SNC at $\vec{x}$. Using the smoothness of the function $s$ in sum (4.20), we can show that relation (4.4) with $x_{1}^{*} \in D^{*} F(\bar{x}, \bar{y})\left(y^{*}\right)$ and $x_{2}^{*} \in N(\bar{x} ; \Omega)$ therein is equivalent to

$$
0=\sum_{i=0}^{m} \lambda_{i} \nabla s_{i}(\bar{x})+x^{*}+\widetilde{x}^{*}, \quad\left(\lambda_{0}, \ldots, \lambda_{m+r}, \widetilde{x}^{*}\right) \neq 0
$$

with $x^{*} \in D^{*} f(\bar{x})\left(\lambda_{m+1}, \ldots, \lambda_{m+r}\right), \widetilde{x}^{*} \in N(\bar{x} ; \Omega)$, and $\lambda_{0} \geq 0$. Recalling that $\nabla s_{i}(\bar{x})=x_{i}^{*}$ for $i=0, \ldots, m$, we arrive at (4.17). To derive (4.18) from (4.17) when $\varphi_{i}$ are locally Lipschitzian for $i=m+1, \ldots, m+r$, it is sufficient to observe that $f$ is automatically SNC at $\bar{x}$ in this case and then to apply the (common) scalarization formula in (2.8) to the coderivative $D^{*} f(\bar{x})$, which gives

$$
D^{*} f(\bar{x})\left(\lambda_{m+1}, \ldots, \lambda_{m+r}\right)=\partial\left(\sum_{i=m+1}^{m+r} \dot{\lambda}_{i} \varphi_{i}\right)(\bar{x})
$$

and completes the proof of the theorem. 


\section{Mathematical Programs with Equilibrium Constraints}

In this section we consider a special class of optimization problems known as mathematical programs with equilibrium constraints (MPEC). A characteristic feature of these problems is the presence, among other constraints, "equilibrium constraints" of the type $y \in S(x)$, where $S(x)$ usually represents the solution map to a "lower-level" problem of parametric optimization. MPEC naturally appear in various aspects of hierarchical optimization and equilibrium theory as well as in many practical applications, especially those related to mechanical and economic modeling. We refer the reader to the books $[11,23]$ for systematic expositions, examples, and applications of such problems in finite-dimensional spaces.

A general class of MPEC considered in this section is given in the following abstract form:

$$
\text { minimize } \varphi(x, y) \text { subject to } y \in S(x), x \in \Omega
$$

where $S: X \rightrightarrows Y$ be a set-valued mapping between Banach spaces, $\varphi: X \rightarrow \bar{R}$, and $\Omega \subset X$. Note that this is an optimization problem with respect to both variables $x$ and $y$ although the constraints on them are given in different forms. The crux of the matter is the presence of the equilibrium constraints $y \in S(x)$ on the decision variable $y$, where the sets $S(x)$ typically describe the so-called solution maps to parametric variational inequalities and complementarity problems of various types.

Our main attention is paid to the case when the equilibrium map $S$ is given in the form

$$
S(x):=\{y \in Y \mid 0 \in f(x, y)+Q(x, y)\}
$$

with $f: X \times Y \rightarrow Z$ and $Q: X \times Y \rightrightarrows Z$, i.e., $S$ describes solution maps to the parametric variational systems/generalized equations defined by

$$
0 \in f(x, y)+Q(x, y)
$$

Such a model covers solution maps to the classical variational inequalities and complementarity problems as well as to their various extensions and modifications. We refer the reader to $[22,27,28]$ and the bibliographies therein for first-order necessary optimality conditions obtained for important special cases of finite-dimensional MPEC problems of type (5.1), (5.2) that particularly involve basic normals, subgradients, and coderivatives of the initial data. In what follows we derive new optimality conditions in both lower and upper subdifferential forms for general MPEC problems and some of their specifications.

Let us first consider problem (5.1). It can be reduced to the standard form (3.4) with two geometric constraints given in spaces with product structures. Based on Theorem 3.2, we derive the two types of subdifferential optimality conditions of the normal type under mild constraint qualifications involving the mixed coderivative of $S$. For simplicity we assume the Lipschitz continuity of the cost function $\varphi$ in the case of lower subdifferential conditions. Note again that in all the presented results the SNC/PSNC assumptions are automatic if the spaces in question are finite-dimensional.

Theorem 5.1 (upper and lower subdifferential conditions for abstract MPEC). Let $(\bar{x}, \bar{y}$ ) be a local optimal solution to problem (5.1). Assume that the spaces $X$ and $Y$ are Asplund and that 
the sets $\operatorname{gph} S$ and $\Omega$ are locally closed around $(\bar{x}, \bar{y})$ and $\bar{x}$, respectively. Assume also that either $S$ is PSNC at $(\bar{x}, \bar{y})$ or $\Omega$ is SNC at $\bar{x}$, and that the mixed qualification condition

$$
D_{M}^{*} S(\bar{x}, \bar{y})(0) \cap(-N(\bar{x} ; \Omega))=\{0\}
$$

is fulfilled. Then one has

$$
-x^{*} \in D_{N}^{*} S(\bar{x}, \bar{y})\left(y^{*}\right)+N(\bar{x} ; \Omega)
$$

for every $\left(x^{*}, y^{*}\right) \in \widehat{\partial}^{+} \varphi(\bar{x}, \bar{y})$. In in addition $\varphi$ is supposed to be Lipschitz continuous around $(\bar{x}, \bar{y})$, then there is $\left(x^{*}, y^{*}\right) \in \partial \varphi(\bar{x}, \bar{y})$ such that $(5.4)$ holds.

Proof. Observe that $\bar{z}=(\bar{x}, \bar{y})$ provides a local minimum to the function $\varphi$ subject to the constraints $z=(x, y) \in \Omega_{1}:=\operatorname{gph} S$ and $z \in \Omega_{2}:=\Omega \times Y$ in the Asplund space $X \times Y$. Applying the upper subdifferential conditions of Theorem 3.2(i) to the latter problem, one can easily see that the PSNC property of $\Omega_{1}$ at $\bar{z}$ with respect to $X$ reduces to the PSNC property of the mapping $S$ at this point, and that $\Omega_{2}$ is always strongly PSNC at $\bar{z}$ with respect to $Y$ being also SNC at this point if and only if $\Omega$ is SNC at $\bar{x}$. Moreover, the mixed qualification condition (5.3) clearly implies that the set system $\left\{\Omega_{1}, \Omega_{2}\right\}$ satisfies the limiting qualification condition (3.5) at $\bar{z}$. Thus we have, by Theorem $3.2(\mathrm{i})$, that

$$
-\widehat{\partial}^{+} \varphi(\bar{x}, \bar{y}) \subset N((\bar{x}, \bar{y}) ; \operatorname{gph} S)+N(\bar{x} ; \Omega) \times\{0\},
$$

which surely implies the upper subdifferential condition (5.4) for every $\left(x^{*}, y^{*}\right) \in \widehat{\partial}^{+} \varphi(\bar{x}, \bar{y})$.

If $\varphi$ is additionally assumed to be locally Lipschitzian around $(\bar{x}, \bar{y})$, it is automatically SNEC at this point and the qualification condition (3.7) holds. This we have

$$
(0,0) \in \partial \varphi(\bar{x}, \bar{y})+N((\bar{x}, \bar{y}) ; \operatorname{gph} S)+N(\bar{x} ; \Omega) \times\{0\}
$$

by Theorem 3.2(ii), which implies (5.4) with some $\left(x^{*}, y^{*}\right) \in \partial \varphi(\bar{x}, \bar{y})$.

Observe that, due to [13, Theorem 3.3], the equilibrium constraint map $S$ is PSNC at $(\bar{x}, \bar{y})$ and the mixed qualification condition (5.3) automatically holds if $S$ satisfies the Aubin Lipschitz-like (or "pseudo-Lipschitzian") property, which therefore is a constraint qualification ensuring the normal form of both lower and upper subdifferential optimality conditions for general MPEC. The reader can find efficient conditions for the Lipschitz-like property of variational systems (5.2) and their specifications in $[16,22,27,28]$ and the references therein.

Note also that the optimality conditions in the normal form of Theorem 5.1 easily imply the ones in the non-qualified (Fritz John) form with no constraint qualification (5.3). In the case of upper subdifferential conditions we have $\lambda \in\{0,1\}$ such that for every $\left(x^{*}, y^{*}\right) \in \widehat{\partial}^{+} \varphi(\bar{x}, \bar{y})$ there exist $x_{1}^{*} \in D_{N}^{*} S(\bar{x}, \bar{y})\left(y^{*}\right)$ and $x_{2}^{*} \in N(\bar{x} ; \Omega)$ satisfying

$$
\lambda x^{*}+x_{1}^{*}+x_{2}^{*}=0, \quad\left(\lambda, x_{1}^{*}\right) \neq 0,
$$

provided that either $S$ is PSNC at $(\bar{x}, \bar{y})$ or $\Omega$ is SNC at $\bar{x}$. Indeed, (5.5) reduces to (5.4) with $\lambda=1$ when the constraint qualification (5.3) is imposed. The negation of (5.3) implies (5.5) with $\lambda=0$, since $D_{M}^{*} S(\bar{x}, \bar{y}) \subset D_{N}^{*} S(\bar{x}, \bar{y})$. Similarly, (5.5) gives a non-qualified lower subdifferential 
condition with some $\left(x^{*}, y^{*}\right) \in \partial \varphi(\bar{x}, \bar{y})$ when $\varphi$ is locally Lipschitzian. In what follows we present only normal/qualified conditions for MPEC problems.

Next let us consider a general class of MPEC problems with equilibrium constraints governed by parameter-dependent variational systems of type (5.2), i.e., the MPEC given by:

$$
\text { minimize } \varphi(x, y) \text { subject to } 0 \in f(x, y)+Q(x, y), \quad x \in \Omega \text {. }
$$

Based on Theorem 5.1, we derive both upper and lower subdifferential conditions for problem (5.6) employing recent results of [16] on computing and estimating coderivatives of solution maps (5.2) together with SNC calculus rules in infinite dimensions.

Theorem 5.2 (upper and lower subdifferential conditions for MPEC with general variational constraints). Let $(\bar{x}, \bar{y})$ be a local optimal solution to (5.6), where $f: X \times Y \rightarrow Z$ and $Q: X \times Y \rightarrow Z$ are mappings between Asplund spaces. Assume that $f$ is continuous around $(\bar{x}, \bar{y})$, that $\Omega$ is locally closed around $\bar{x}$, and that the graph of $Q$ is locally closed around $(\bar{x}, \bar{y}, \bar{z})$ with $\bar{z}:=-f(\bar{x}, \bar{y})$. Suppose also that one of the following assumptions (a)-(c) holds:

(a) $\Omega$ and $Q$ are $S N C$ at $\bar{x}$ and $(\bar{x}, \bar{y}, \bar{z})$, respectively, and the two qualification conditions

$$
\begin{gathered}
{\left[\left(x^{*}, 0\right) \in D_{N}^{*} f(\bar{x}, \bar{y})\left(z^{*}\right)+D_{N}^{*} Q(\bar{x}, \bar{y}, \bar{z})\left(z^{*}\right),-x^{*} \in N(\bar{x} ; \Omega)\right] \Longrightarrow x^{*}=0,} \\
{\left[\left(x^{*}, y^{*}\right) \in D_{N}^{*} f(\bar{x}, \bar{y})\left(z^{*}\right) \cap\left(-D_{N}^{*} Q(\bar{x}, \bar{y}, \bar{z})\left(z^{*}\right)\right)\right] \Longrightarrow x^{*}=y^{*}=z^{*}=0}
\end{gathered}
$$

are satisfied; the latter is equivalent to

$$
\left[0 \in \partial\left\langle z^{*}, f\right\rangle(\bar{x}, \bar{y})+D_{N}^{*} Q(\bar{x}, \bar{y}, \bar{z})\left(z^{*}\right)\right] \Longrightarrow z^{*}=0
$$

when $f$ is strictly Lipschitzian around $(\bar{x}, \bar{y})$.

(b) $\Omega$ is SNC at $\bar{x}, \operatorname{dim} Z<\infty, f$ is Lipschitz continuous around $(\bar{x}, \bar{y})$, and the qualification conditions

$$
\left[\left(x^{*}, 0\right) \in \partial\left\langle z^{*}, f\right\rangle(\bar{x}, \bar{y})+D_{N}^{*} Q(\bar{x}, \bar{y}, \bar{z})\left(z^{*}\right), \quad-x^{*} \in N(\bar{x} ; \Omega)\right] \Longrightarrow x^{*}=0
$$

and (5.9) are satisfied.

(c) $Q$ is SNC at $(\bar{x}, \bar{y}, \bar{z}), f$ is PSNC at $(\bar{x}, \bar{y})$ (which is automatic when it is Lipschitz continuous around this point), and the qualification conditions (5.7) and (5.8) hold.

Then for every $\left(x^{*}, y^{*}\right) \in \widehat{\partial}^{+} \varphi(\bar{x}, \bar{y})$ there are $\widetilde{x}^{*} \in N(\bar{x} ; \Omega)$ and $z^{*} \in Z^{*}$ such that

$$
\left(-x^{*}-\widetilde{x}^{*},-y^{*}\right) \in D_{N}^{*} f(\bar{x}, \bar{y})\left(z^{*}\right)+D_{N}^{*} Q(\bar{x}, \bar{y}, \bar{z})\left(z^{*}\right)
$$

If in addition $\varphi$ is Lipschitz continuous around $(\bar{x}, \bar{y})$, then (5.10) is satisfied for some $z^{*} \cdot \in Z^{*}$, $\left(x^{*}, y^{*}\right) \in \partial \varphi(\bar{x}, \bar{y})$, and $\widetilde{x}^{*} \in N(\bar{x} ; \Omega)$.

Proof. Let us apply the upper subdifferential optimality conditions from Theorem 5.1 to problem (5.6), i.e., in the case when the equilibrium constraints $y \in S(x)$ are given in the variational/generalized equation form (5.2). It is easy to see that the continuity and closedness assumptions made on $f$ and $Q$ ensure the local closedness of $S$. To proceed further, we first assume 
that $\Omega$ is SNC at $\bar{x}$ and use the coderivative upper estimate for such mappings $S$ obtained in [16, Theorem 4.1]. This gives the inclusion

$$
\begin{aligned}
D_{N}^{*} S(\bar{x}, \bar{y})\left(y^{*}\right) \subset \quad & \left\{x^{*} \in X^{*} \mid \exists z^{*} \in Z^{*}\right. \text { with } \\
& \left.\left(x^{*},-y^{*}\right) \in D_{N}^{*} f(\bar{x}, \bar{y})\left(z^{*}\right)+D_{N}^{*} Q(\bar{x}, \bar{y}, \bar{z})\left(z^{*}\right)\right\}
\end{aligned}
$$

under the qualification condition (5.8) and the assumptions on either $Q$ made in (a) or on $f$ and $Z$ made in (b). Then substituting (5.11) into (5.3) and (5.4), we arrive at at the upper subdifferential optimality conditions of the theorem under the assumptions made in either (a) or (b).

Now we consider the remaining case when $S$ is PSNC in Theorem 5.1 and provide efficient conditions in terms of $f$ and $Q$ ensuring the latter (even SNC) property for the equilibrium map $S$ given in (5.2). Observe that the graph of $S$ is represented as the inverse image

$$
\operatorname{gph} S=g^{-1}(\operatorname{gph} Q) \text { with } g(x, y):=(x, y,-f(x, y)) \text {. }
$$

Applying [20, Theorem 3.8] in this setting, we check that the graph of $S$ is SNC at $(\bar{x}, \bar{y})$ if the qualification condition (5.8) is satisfied and the mapping $g$ in (5.12) is PSNC at $(\bar{x}, \bar{y})$. Let us show that the latter is equivalent to the PSNC property of $f$ at this point in the Asplund space setting. Indeed, taking sequences $\left(x_{k}^{*}, y_{k}^{*}\right) \in \widehat{D}^{*} g\left(x_{k}, y_{k}\right)\left(u_{k}^{*}, v_{k}^{*}, z_{k}^{*}\right)$ with $\left(x_{k}^{*}, y_{k}^{*}\right) \stackrel{w^{*}}{\rightarrow}(0,0)$ and $\left\|\left(u_{k}^{*}, v_{k}^{*}, z_{k}^{*}\right)\right\| \rightarrow 0$, we get

$$
\left(x_{k}^{*}, y_{k}^{*}\right)=\left(u_{k}^{*}, v_{k}^{*}\right)+\left(\hat{x}_{k}^{*}, \hat{y}_{k}^{*}\right) . \text { with }\left(\hat{x}_{k}^{*}, \hat{y}_{k}^{*}\right) \in \widehat{D}^{*} f\left(x_{k}, y_{k}\right)\left(-z_{k}^{*}\right)
$$

due to the representation

$$
g(x, y)=(x, y, 0)+(0,0,-f(x, y))
$$

and the elementary equality rule for representing $\widehat{D}^{*} g\left(x_{k}, y_{k}\right)$ in the above sum. This implies that $\left(\hat{x}_{k}^{*}, \hat{y}_{k}^{*}\right) \stackrel{w^{*}}{\rightarrow}(0,0)$, and hence $\left\|\left(\hat{x}_{k}^{*}, \hat{y}_{k}^{*}\right)\right\| \rightarrow 0$ by the PSNC property of $f$. Thus $\left\|\left(x_{k}^{*}, y_{k}^{*}\right)\right\| \rightarrow 0$ as well, i.e., $g$ is PSNC at $(\bar{x}, \bar{y})$. This ends the proof of the upper subdifferential part in the theorem.

The last (lower subdifferential) statement of the theorem follows from the lower subdifferential result of Theorem 5.1 by the above arguments.

In MPEC problems most interesting for the theory and applications, equilibrium/variational constraints are usually defined via first-order subdifferentials of extended-real-valued functions. In particular, the classical equilibrium constraints given by parametric variational inequalities and complementarity conditions are naturally defined in terms of subgradients and normals for convex functions and sets. Let us consider a broader class of such MPEC with equilibrium constraints defined via the basic subdifferential (2.3) of composite functions with no convexity assumptions:

$$
\text { minimize } \varphi(x, y) \text { subject to } 0 \in f(x, y)+\partial(\psi \circ g)(x, y), \quad x \in \Omega \text {, }
$$

where $f: X \times Y \rightarrow X^{*} \times Y^{*}$ and $g: X \times Y \rightarrow W$ are single-valued mappings between Banach spaces, and where $\psi: W \rightarrow \overline{\mathbb{R}}$ is an extended-real-valued function. The MPEC problem (5.13) is a special case of (5.6) with the subdifferential set-valued mapping $Q(x, y)=\partial(\psi \circ g)(x, y)$. Since coderivatives of first-order subdifferential mappings define second-order subdifferentials as in (2.10), 
one can therefore deduce necessary optimality conditions for (5.13) from the ones for (5.6) obtained in Theorem 5.2 using second-order subdifferential chain rules. Let us present some upper and lower subdifferential optimality conditions obtained in this way for MPEC problems of type (5.13). First we consider the case of smooth and parameter-independent mappings $g: Y \rightarrow W$ in (5.13) with surjective derivatives in infinite-dimensional settings.

Theorem 5.3 (optimality conditions for MPEC governed by parameter-independent generalized variational inequalities). Let $(\bar{x}, \bar{y})$ be a local optimal solution to problem (5.13). with $f: X \rightarrow Y, g: Y \rightarrow W$, and $\psi: W \rightarrow \overline{\mathbb{R}}$. Suppose that $W$ is Banach, $X$ is Asplund, $Y$ is finite-dimensional and that the following assumptions hold:

(a) $f: X \times Y \rightarrow Y^{*}$ is strictly differentiable at $(\bar{x}, \bar{y})$ with the surjective partial derivative $\nabla_{x} f(\bar{x}, \bar{y}): X \rightarrow Y^{*}$.

(b) $g$ is continuously differentiable around $\bar{y}$ with the surjective derivative $\nabla g(\bar{y}): Y \rightarrow W$, and the mapping $\nabla g: Y \rightarrow \mathcal{L}(Y, W)$ into the space of linear bounded operators from $Y$ to $W$ is strictly differentiable at $\bar{y}$.

(c) $\Omega$ is locally closed around $\vec{x}$ and the graph of $\partial \psi$ is locally closed around $(\bar{w}, \bar{v})$, where $\bar{w}:=g(\bar{y})$ and where $\bar{v} \in W^{*}$ is a unique functional satisfying the relations

$$
-f(\bar{x}, \bar{y})=\nabla g(\bar{y})^{*} \bar{v}, \quad \bar{v} \in \partial \psi(\bar{w}) .
$$

Then for every $\left(x^{*}, y^{*}\right) \in \widehat{\partial}^{+} \varphi(\bar{x}, \bar{y})$ there is $u \in Y$ such that

$$
\begin{aligned}
& -x^{*} \in \nabla_{x} f(\bar{x}, \bar{y})^{*} u+N(\bar{x} ; \Omega) \quad \text { and } \\
& -y^{*} \in \nabla_{y} f(\bar{x}, \bar{y})^{*} u+\nabla^{2}\langle\bar{v}, g\rangle(\bar{y})^{*} u+\nabla g(\bar{y})^{*} \partial_{N}^{2} \psi(\bar{w}, \bar{v})(\nabla g(\bar{y}) u)
\end{aligned}
$$

provided that $u=0$ is the only vector satisfying the system of inclusions

$$
\left\{\begin{array}{l}
0 \in \nabla_{x} f(\bar{x}, \bar{y})^{*} u+N(\bar{x} ; \Omega), \\
0 \in \nabla_{y} f(\bar{x}, \bar{y})^{*} u+\nabla^{2}\langle\bar{v}, g\rangle(\bar{y})^{*} u+\nabla g(\bar{y})^{*} \partial_{N}^{2} \psi(\bar{w}, \bar{v})(\nabla g(\bar{y}) u) .
\end{array}\right.
$$

In in addition $\varphi$ is locally Lipschitzian around $(\bar{x}, \bar{y})$, then there are $u \in Y$ and $\left(x^{*}, y^{*}\right) \in \partial \varphi(\bar{x}, \bar{y})$ satisfying (5.14).

Proof. To establish the upper subdifferential conditions of the theorem, we employ the results of Theorem 5.2 under the assumptions in (c) for $Q(y):=\partial(\psi \circ g)(y)$. Taking into account the strict differentiability of $f$ at $(\bar{x}, \bar{y})$ with the surjectivity of $\nabla_{x} f(\bar{x}, \bar{y})$ and the parameter-independence of $Q$, one has the qualification condition (5.8) automatically fulfilled, while (5.7) reduced to

$$
\left[0 \in \nabla_{x} f(\bar{x}, \bar{y})^{*} u+N(\bar{x} ; \Omega), 0 \in \nabla_{y} f(\bar{x}, \bar{y})^{*} u+\partial^{2}(\psi \circ g)(\bar{y}, \bar{z})(u)\right] \Rightarrow u=0
$$

with $\bar{z}:=-f(\bar{x}, \bar{y})$ provided that the mapping $\partial(\psi \circ g)(\cdot)$ is locally closed-graph around $(\bar{y}, \bar{z})$. Observe the SNC property of $Q$ and PSNC property of $f$ at the reference points follow immediately from the the finite dimensionality of $Y$ and the strict differentiability of $f$. Then, by the superdifferential optimality condition of Theorem 5.2 applying to (5.13), for every $\left(x^{*}, y^{*}\right) \in \hat{\partial}^{+} \varphi(\bar{x}, \bar{y})$ there is $u \in Y$ such that

$$
-x^{*} \in \nabla_{x} f(\bar{x}, \bar{y})^{*} u+N(\bar{x} ; \Omega), \quad-y^{*} \in \nabla_{y} f(\bar{x}, \bar{y})^{*} u+\partial^{2}(\psi \circ g)(\bar{y}, \bar{z})(u) .
$$


Using now the first-order subdifferential chain rule of [21, Corollary 3.11] in the case of inner mappings $g$ with the surjective derivative at $\bar{y}$ (and hence at $y$ near $\bar{y}$ ), we have the equality

$$
\partial(\psi \circ g)(y)=\nabla g(y)^{*} \partial \psi(w)
$$

for all $y$ close to $\breve{y}$ and $w=g(y)$, which implies that the graph of $\partial(\psi \circ g)(\cdot)$ is locally closed around $(\bar{y}, \bar{z})$ if and only if the subdifferential mapping $\partial \psi(\cdot)$ is closed-graph around $(\bar{w}, \bar{v})$. Applying further the second-order subdifferential chain rule of $\left[15\right.$, Theorem 4.1] to $\partial^{2}(\psi \circ g)(\bar{y}, \bar{z})$ and taking into account that $\nabla g(\bar{y})^{* *}=\nabla g(\bar{y})$ under the assumptions made, one has

$$
\partial_{N}^{2}(\psi \circ g)(\bar{y}, \bar{z})(u)=\nabla^{2}\langle\bar{v}, g\rangle(\bar{y})^{*} u+\nabla g(\bar{y})^{*} \partial_{N}^{2} \psi(\bar{w}, \bar{v})(\nabla g(\bar{y}) u) .
$$

Substituting this into the above relationships, we arrive at the upper subdifferential conditions stated in the theorem. If $\varphi$ is locally Lipschitzian around $(\bar{x}, \vec{y})$, the lower subdifferential result of the theorem is deduced by a similar way from the one in Theorem 5.2 .

Note that the closed-graph assumption on $\partial \psi$ in the above theorem automatically holds for continuous functions $\psi$. It also holds for the so-called amenable functions, which play a major role in finite-dimensional variational analysis and optimization; see [25].

Recall that a function $\phi: X \rightarrow \overline{\mathbb{R}}$ is amenable at $\bar{x}$ if there is a neighborhood $U$ of $\bar{x}$ on which $\psi$ can be represented in the composition form $\phi=\psi \circ g$ with a $\mathcal{C}^{1}$ mapping $g: U \rightarrow \mathbb{R}^{m}$ and a proper l.s.c. convex function $\psi: \mathbb{R}^{m} \rightarrow \overline{\mathbb{R}}$ satisfying the qualification condition

$$
\partial \psi^{\infty}(g(\bar{x})) \cap \operatorname{ker} \nabla g(\bar{x})^{*}=\{0\} .
$$

It is strongly amenable at $\bar{x}$ if such a representation exists with $g$ not just $\mathcal{C}^{1}$ but $\mathcal{C}^{2}$. Our next theorem contains upper and lower subdifferential optimality conditions for MPEC (5.13) with parameter-dependent potentials $\phi(x, y):=(\psi \circ g)(x, y)$ given by strongly amenable functions.

Theorem 5.4 (optimality conditions for MPEC with parameter-dependent amenable potentials). Let $(\bar{x}, \bar{y})$ be a local optimal solution to problem (5.13) in finite-dimensional spaces. Assume that $\Omega \subset \mathbb{R}^{n}$ is locally closed around $\bar{x}$, that $f: \mathbb{R}^{n} \times \mathbb{R}^{m} \rightarrow \mathbb{R}^{n} \times \mathbb{R}^{m}$ is continuous around $(\bar{x}, \bar{y})$, and that $\phi=\psi \circ g$ is strongly amenable at this point with $g: \mathbb{R}^{n} \times \mathbb{R}^{m} \rightarrow \mathbb{R}^{l}$. Denote $\bar{w}:=g(\bar{x}, \bar{y}), \bar{z}:=-f(\bar{x}, \bar{y}) \in \partial(\psi \circ g)(\bar{x}, \bar{y})$,

$$
M(\bar{x}, \bar{y}):=\left\{\bar{v} \in \mathbb{R}^{l} \mid v \in \partial \psi(\bar{w}), \nabla g(\bar{x}, \bar{y})^{*} \bar{v}=\bar{z}\right\}
$$

and impose the following second-order qualification conditions:

$$
\begin{gathered}
\partial^{2} \psi(\bar{w}, \bar{v})(0) \cap \operatorname{ker} \nabla g(\bar{x}, \bar{y})^{*}=\{0\} \text { for all } \bar{v} \in M(\bar{x}, \bar{y}), \\
(p, q) \in \bigcup_{\bar{v} \in M(\bar{x}, \bar{y})}\left[\nabla^{2}\langle\bar{v}, g\rangle(\bar{x}, \bar{y}) u+\nabla g(\bar{x}, \bar{y})^{*} \partial^{2} \psi(\bar{w}, \bar{v})(\nabla g(\bar{x}, \bar{y}) u)\right] \\
\bigcap\left[-D^{*} f(\bar{x}, \bar{y})(u)\right] \Longrightarrow(p, q, u)=(0,0,0), \\
{\left[\left(x^{*}, 0\right) \in D^{*} f(\bar{x}, \bar{y})(u)+\bigcup_{\bar{v} \in M(\bar{x}, \bar{y})}\left[\nabla^{2}\langle\bar{v}, g\rangle(\bar{x}, \bar{y})(u)\right.\right.} \\
\left.\left.+\nabla g(\bar{x}, \bar{y})^{*} \partial^{2} \psi(\bar{w}, \bar{v})(\nabla g(\bar{x}, \bar{y}) u)\right],-x^{*} \in N(\bar{x} ; \Omega)\right] \Longrightarrow x^{*}=0 .
\end{gathered}
$$


Then for every $\left(x^{*}, y^{*}\right) \in \widehat{\partial}^{+} \varphi(\bar{x}, \bar{y})$ there is $u \in \mathbb{R}^{n} \times \mathbb{R}^{m}$ such that

$$
\begin{aligned}
\left(-x^{*},-y^{*}\right) \in & D^{*} f(\bar{x}, \bar{y})(u)+\bigcup_{\bar{v} \in M(\bar{x}, \bar{y})}\left[\nabla^{2}\langle\bar{v}, g\rangle(\bar{x}, \bar{y})(u)\right. \\
& \left.+\nabla g(\bar{x}, \bar{y})^{*} \partial^{2} \psi(\bar{w}, \bar{v})(\nabla g(\bar{x}, \bar{y}) u)\right]+N(\bar{x} ; \Omega) .
\end{aligned}
$$

If in addition $\varphi$ is Lipschitz continuous around $(\bar{x}, \bar{y})$, then there are subgradients $\left(x^{*}, y^{*}\right) \in \partial \varphi(\bar{x}, \bar{y})$ satisfying (5.16) with some vector $u \in \mathbb{R}^{n} \times \mathbb{R}^{m}$.

Proof. It is sufficient to justify the upper subdifferential part of the theorem, because the proof of the lower subdifferential part is similar. We apply Theorem 5.2 for $Q(x, y)=\partial(\psi \circ g)$ under the assumptions in (a) in the finite-dimensional setting. Since

$$
D^{*} Q(\bar{x}, \bar{y}, \bar{z})=\partial^{2}(\psi \circ g)(\bar{x}, \bar{y}, \bar{z})
$$

one may employ the second-order subdifferential chain rule for $\psi \circ g$ from [15, Theorem 4.2(ii)], which is available under the assumptions made (and even in more general infinite-dimensional settings). Using the cited theorem (actually its Corollary 4.3), we have the inclusion

$$
\partial^{2}(\psi \circ g)(\bar{x}, \bar{y}, \bar{z})(u) \subset \bigcup_{\bar{v} \in M(\bar{x}, \bar{y})}\left[\nabla^{2}\langle\bar{v}, g\rangle(\bar{x}, \bar{y})^{*} u+\nabla g(\bar{x}, \bar{y})^{*} \partial^{2} \psi(\bar{w}, \bar{v})(\nabla g(\bar{x}, \bar{y}) u)\right] .
$$

Substituting this into the corresponding relationships of Theorem 5.2 with the coderivative expression (5.17), we arrive at the conclusions of the theorem.

Observe that the qualification condition (5.15) reduces to

$$
0 \in \partial\langle u, f\rangle(\bar{x}, \bar{y})+\bigcup_{\bar{v} \in M(\bar{x}, \bar{y})}\left[\nabla^{2}\langle\bar{v}, g\rangle(\bar{x}, \bar{y})^{*} u+\nabla g(\bar{x}, \bar{y})^{*} \partial^{2} \psi(\bar{w}, \bar{v})(\nabla g(\bar{x}, \bar{y}) u)\right] \Longrightarrow u=0
$$

when $f$ is locally Lipschitzian around $(\bar{x}, \bar{y})$. It holds automatically if $g=g(y)$ and $f$ is strictly differentiable at $(\bar{x}, \bar{y})$ with the surjective partial derivative $\nabla_{x} f(\bar{x}, \bar{y})$.

Finally in this paper we consider a class of MPEC problems with equilibrium constraints involving another type of subdifferential compositions, namely:

$$
\text { minimize } \varphi(x, y) \text { subject to } 0 \in f(x, y)+(\partial \psi \circ g)(x, y), \quad x \in \Omega \text {, }
$$

where $g: X \times Y \rightarrow W, \psi: W \rightarrow \overline{\mathbb{R}}$, and $f: X \times Y \rightarrow W^{*}$. One can see that the main difference between the two composite forms in (5.13) and (5.18) is that the former involves the first-order subdifferential of the composite potential $\psi \circ g$, while the generalized equation in (5.18) contains a composition in its field/set-valued part $Q(x, y)=(\partial \psi \circ g)(x, y)$. Systems of the latter type frequently arise, e.g., in the modeling of mechanical and economic equilibria and cover, in particular, parameter-dependent implicit complementarity problems [22]: given $x \in \mathbb{R}^{n}$, find $y \in \mathbb{R}^{m}$ satisfying

$$
f(x, y) \geq 0, \quad y-g(x, y) \geq 0, \quad\langle f(x, y), y-g(x, y)\rangle=0 .
$$

The standard nonlinear complementarity problem corresponds to (5.19) with $g=0$. Our next theorem contains general necessary optimality conditions in the upper and lower subdifferential forms for infinite-dimensional MPEC of type (5.18). 
Theorem 5.5 (optimality conditions for general MPEC with composite fields). Let $(\bar{x}, \bar{y})$ be a local optimal solution to problem (5.18) with $\Omega$ closed around $\bar{x}, \bar{w}:=g(\bar{x}, \bar{y})$, and $\bar{z}:=-f(\bar{x}, \bar{y})$. The following assertions hold:

(i) Assume that $X, Y$ are Asplund while $W$ is Banach, that $g=g(y)$ is strictly differentiable at $\bar{y}$ with the surjective derivative $\nabla g(\bar{y})$, that $f$ is strictly differentiable at $(\bar{x}, \bar{y})$ with the surjective partial derivative $\nabla_{x} f(\bar{x}, \bar{y})$, and that $u=0 \in W^{* *}$ is the only element satisfying

$$
0 \in \nabla_{x} f(\bar{x}, \bar{y})^{*} u+N(\bar{x} ; \Omega), \quad 0 \in \nabla_{y} f(\bar{x}, \bar{y})^{*} u+\nabla g(\bar{y})^{*} \partial_{N}^{2} \psi(\bar{w}, \bar{z})(u) .
$$

Then for every $\left(x^{*}, y^{*}\right) \in \widehat{\partial}^{+} \varphi(\bar{x}, \bar{y})$ there is $u \in W^{* *}$ such that

$$
\begin{aligned}
& -x^{*} \in \nabla_{x} f(\bar{x}, \bar{y})^{*} u+N(\bar{x} ; \Omega), \\
& -y^{*} \in \nabla_{y} f(\bar{x}, \bar{y})^{*} u+\nabla g(\bar{y})^{*} \partial_{N}^{2} \psi(\bar{w}, \bar{z})(u)
\end{aligned}
$$

provided that either $\Omega$ is $S N C$ at $\bar{x}$ or $\partial \psi$ is $S N C$ at $(\bar{w}, \bar{z})$.

(ii) Assume that $X, Y, W, W^{*}$ are Asplund, that $f$ and $g$ are continuous around $(\bar{x}, \bar{y})$, that the graph of $\partial \psi$ is norm-closed around $(\bar{w}, \bar{z})$, that

$$
\partial_{N}^{2} \psi(\bar{w}, \bar{z})(0) \cap \operatorname{ker} D_{N}^{*} g(\bar{x}, \bar{y})=\{0\}
$$

that $x^{*}=0$ is the only element satisfying

$$
\left(x^{*}, 0\right) \in D_{N}^{*} f(\bar{x}, \bar{y})(u)+D_{N}^{*} g(\bar{x}, \bar{y}) \circ \partial_{N}^{2} \psi(\bar{w}, \bar{z})(u),-x^{*} \in N(\bar{x} ; \Omega)
$$

for some $u \in W^{* *}$, and that $\left(x^{*}, y^{*}, u\right)=(0,0,0)$ is the only one satisfying

$$
\left(x^{*}, y^{*}\right) \in D_{N}^{*} f(\bar{x}, \bar{y})(u) \cap\left(-D_{N}^{*} g(\bar{x}, \bar{y}) \circ \partial_{N}^{2} \psi(\bar{w}, \bar{z})(u)\right) .
$$

Then for every supergradient $\left(x^{*}, y^{*}\right) \in \widehat{\partial}^{+} \varphi(\bar{x}, \bar{y})$ there are $\widetilde{x}^{*} \in N(\bar{x} ; \Omega)$ and $u \in W^{* *}$ such that

$$
\left(-x^{*}-\widetilde{x}^{*},-y^{*}\right) \in D_{N}^{*} f(\bar{x}, \bar{y})(u)+D_{N}^{*} g(\bar{x}, \bar{y}) \circ \partial_{N}^{2} \psi(\bar{w}, \bar{z})(u)
$$

provided that either $f$ is Lipschitz continuous around $(\bar{x}, \bar{y})$ and $\operatorname{dim} W<\infty$, or $g$ is PSNC at $(\bar{x}, \bar{y})$ and $\partial \psi$ is SNC at $(\bar{w}, \bar{z})$, or $g$ is SNC at $(\bar{x}, \bar{y})$ and $\partial \psi^{-1}$ is PSNC at $(\bar{z}, \bar{w})$.

(iii) Assume that $\varphi$ is Lipschitz continuous around $(\bar{x}, \bar{y})$ in addition to the assumptions in either (i) or (ii). Then there are, respectively, $\left(x^{*}, y^{*}\right) \in \partial \varphi(\vec{x}, \vec{y})$ and $u \in W^{* *}$ satisfying (5.20) and $\left(x^{*}, y^{*}\right) \in \partial \varphi(\bar{x}, \bar{y}), \widetilde{x}^{*} \in N(\bar{x} ; \Omega), u \in W^{* *}$ satisfying $(5.21)$.

Proof. To justify (i), we employ the upper subdifferential result of Theorem 5.1 with the equilibrium constraints given by

$$
S(x):=\{y \in Y \mid 0 \in f(x, y)+(\partial \psi \circ g)(x, y)\} .
$$

Since one obviously has

$$
\operatorname{gph} S=\{(x, y) \in X \times Y \mid h(x, y) \in \operatorname{gph}(\partial \psi \circ g)\} \text { with } h(x, y):=(y,-f(x, y))
$$

and $\nabla h(\bar{x}, \bar{y})$ is surjective if and only if $\nabla_{x} f(\bar{x}, \bar{y})$ is, it follows from [21, Corollary 3.9] that

$$
\begin{aligned}
D_{N}^{*} S(\bar{x}, \bar{y})\left(y^{*}\right)=\left\{x^{*} \in X^{*} \mid \quad\right. & \exists u \in W^{* *} \text { with } x^{*}=\nabla_{x} f(\bar{x}, \bar{y})^{*} u, \\
& \left.-y^{*} \in \nabla_{y} f(\bar{x}, \bar{y})^{*} u+D_{N}^{*}(\partial \psi \circ g)(\bar{y}, \bar{z})(u)\right\}
\end{aligned}
$$


for any Banach spaces $X, Y, W$. Moreover, Corollary 5.3 of [21] implies that the SNC property of $S$ at $(\bar{x}, \bar{y})$ is equivalent to the one for $\partial \psi \circ g$ at $(\bar{y}), \bar{z})$. In turn, the latter is equivalent to the SNC property of $\partial \psi$ at $(\bar{w}, \bar{z})$ by $[21$, Corollary 5.4], since $\nabla g(\bar{y})$ is assumed to be surjective. To complete the proof of (i), it is sufficient to employ the chain rule

$$
D_{N}^{*}(\partial \psi \circ g)(\bar{y}, \bar{z})(u)=\nabla g(\bar{y})^{*} \partial_{N}^{2} \psi(\bar{w}, \bar{z})(u)
$$

from [21, Theorem 3.10] and substitute it into (5.22).

The proof of assertion (ii) in the Asplund space setting is based on the application of Theorem 5.2 with $Q(x, y)=(\partial \psi \circ g)(x, y)$. The sufficient conditions for the SNC property of the composition $\partial \psi \circ g$ are derived from [20, Theorem 3.8] similarly to the proof of Theorem 5.2. The lower subdifferential conditions in (iii) under the assumptions made follow from Theorems 5.2 and 5.3 by employing the above arguments.

Our final result concerns optimality conditions for MPEC (5.18) in the case of strictly differentiable mappings $f$ and $g$ with possible non-surjective derivatives when the relations of Theorem 5.5 admit essential simplifications.

Corollary 5.6 (optimality conditions for special MPEC with composite fields). Let $(\bar{x}, \bar{y})$ be a local optimal solution to problem (5.18) with $f: X \times Y \rightarrow \mathbb{R}^{m}$ and $g: X \times Y \rightarrow \mathbb{R}^{m}$ strictly differentiable at $(\bar{x}, \bar{y})$ and with $\Omega \subset X$ closed around $\bar{x}$. Assume that $X$ and $Y$ are Asplund, that gph $\partial \psi$ is closed around $(\bar{w}, \bar{z})$ (which is automatic for continuous and amenable functions), that

$$
\partial^{2} \psi(\bar{w}, \bar{z})(0) \cap \operatorname{ker} \nabla g(\bar{x}, \bar{y})^{*}=\{0\} .
$$

and that the system of inclusions

$$
\left\{\begin{array}{l}
x^{*} \in \nabla_{x} f(\bar{x}, \bar{y})^{*} u+\nabla_{x} g(\bar{x}, \bar{y})^{*} \partial^{2} \psi(\bar{w}, \bar{z})(u), \quad-x^{*} \in N(\bar{x} ; \Omega) \\
0 \in \nabla_{y} f(\bar{x}, \bar{y})^{*} u+\nabla_{y} g(\bar{x}, \bar{y})^{*} \partial^{2} \psi(\bar{w}, \bar{z})(u)
\end{array}\right.
$$

has only the trivial solution $x^{*}=u=0$. Then for every upper subgradient $\left(x^{*}, y^{*}\right) \in \widehat{\partial}^{+} \varphi(\bar{x}, \bar{y})$ there is a vector $u \in \mathbb{R}^{m}$ such that

$$
\begin{aligned}
& -x^{*} \in \nabla_{x} f(\bar{x}, \bar{y})^{*} u+\nabla_{x} g(\bar{x}, \bar{y})^{*} \partial^{2} \psi(\bar{w}, \bar{z})(u)+N(\bar{x} ; \Omega), \\
& -y^{*} \in \nabla_{y} f(\bar{x}, \bar{y})^{*} u+\nabla_{y} g(\bar{y})^{*} \partial^{2} \psi(\bar{w}, \bar{z})(u) .
\end{aligned}
$$

If in addition the cost function $\varphi$ is Lipschitz continuous around $(\bar{x}, \bar{y})$, then there are a lower subgradient $\left(x^{*}, y^{*}\right) \in \partial \varphi(\bar{x}, \bar{y})$ and a vector $u \in \mathbb{R}^{m}$ satisfying (5.23).

Proof. This easily follows from assertions (ii) and (iii) of Theorem 5.5 due to the coderivative representation for strictly differentiable mappings; see Section 2.

In the case of finite-dimensional spaces $X$ and $Y$ the lower subdifferential result of Corollary 5.6 is strongly related to the necessary optimality conditions from [22, Theorem 3.1] obtained for a composite MPEC problem of type (5.18) with $\partial \psi$ replaced by a set-valued mapping of closed graph and with geometric constraints on both $x$ and $y$. 
To conclude this paper, we observe that MPEC problems are intrinsically nonsmooth, even in the simplest settings of equilibrium constraints governed by parameter-dependent variational inequalities and complementarity conditions. For models (5.13) and (5.18) this relates to the nonsmoothness of the potential $\psi$, which is actually the indicator (extended-real-valued) function of a convex set for the case of complementarity and standard variational inequality constraints. Practical implementations of the optimality conditions obtained in Theorems 5.3-5.5 require therefore computing/estimating the second-order subdifferentials for attractive classes of nonsmooth functions $\psi$ in (5.13) and (5.18). Efficient calculations of second-order subdifferentials and their applications to special MPEC and related problems are given in $[6,17,22]$ and the references therein. Such calculations and the results obtained above allow us to extend classes of MPEC that can be efficiently handled by generalized differential methods of variational analysis.

Acknowledgments. The author gratefully acknowledges helpful discussions with Jiři Outrata, Terry Rockafellar, and Roger Wets on the subject of this paper.

\section{References}

[1] Aubin, J.-P.: Lipschitz behavior of solutions to convex minimization problems, Math. Oper. Res. 9 (1984), 87-111.

[2] Borwein, J. M. and Strojwas H. M.: Tangential approximations, Nonlinear Anal. 9 (1985), 1347-1366.

[3] Borwein, J. M. and Zhu, Q. J.: A survey of subdifferential calculus with applications, Nonlinear Anal. 38 (1999), 687-773.

[4] Clarke, F. H.: Optimization and Nonsmooth Analysis, Wiley, New York, 1983.

[5] Crandall, M. G., Evans, L. C. and Lions, P.-L.: Some properties of viscosity solutions of the HamiltonJacobi equations, Trans. Amer. Math. Soc. 282 (1984), 487-502.

[6] Dontchev, A. D. and Rockafellar, R. T.: Characterization of strong regularity for variational inequalities over polyhedral convex sets, SIAM J. Optim. 6 (1996), 1087-1105.

[7] Fabian, M. and Mordukhovich, B. S.: Nonsmooth characterizations of Asplund spaces and smooth variational principles, Set-Valued Anal. 6 (1998), 381-406.

[8] Fabian, M. and Mordukhovich, B. S.: Sequential normal compactness versus topological normal compactness in variational analysis, Nonlinear Anal., in press.

[9] Ioffe, A. D.: Necessary conditions in nonsmooth optimization, Math. Oper. Res. 9 (1984), 159-188.

[10] Ioffe, A. D.: Coderivative compactness, metric regularity and subdifferential calculus, In: M. Théra (ed.), Expremental, Constructive, and Nonlinear Analysis, CMS Conference Proc. Vol. 27, American Mathematical Society, Providence, R.I., 2000, pp. 123-164.

[11] Luo, Z. Q., Pang, J. S. and Ralph, D.: Mathematical Programs with Equilibrium Constraints, Cambridge University Press, Cambridge, UK, 1996.

[12] Mordukhovich, B. S.: Approximation Methods in Problems of Optimization and Control, Nauka, Moscow, 1988. 
[13] Mordukhovich, B. S.: Coderivatives of set-valued mappings: calculus and applications, Nonlinear Anal. 30 (1997), 3059-3070.

[14] Mordukhovich, B. S.: The extremal principle and its applications to optimization and economics, In: A. Rubinov and B. Glover (eds.), Optimization and Related Topics, Applied Optimization Vol. 47, Kluwer, Dordrecht, 2001, pp. 343-369.

[15] Mordukhovich, B. S.: Calculus of second-order subdifferentials in infinite dimensions, Control and Cybernetics 31 (2002), No. 3, 557-573.

[16] Mordukhovich, B. S.: Coderivative analysis of variational systems, J. Global Optim., in press.

[17] Mordukhovich, B. S. and Outrata, J. V.: On second-order subdifferentials and their applications, SIAM J. Optim. 12 (2001), 139-169.

[18] Mordukhovich, B. S. and Shao, Y.: Nonsmooth sequential analysis in Asplund spaces, Trans. Amer. Math. Soc. 348 (1996), 1235-1280.

[19] Mordukhovich, B. S. and Wang, B.: Extensions of generalized differential calculus in Asplund spaces, J. Math. Anal. Appl. 272 (2002), 164-186.

[20] Mordukhovich, B. S. and Wang, B.: Calculus of sequential normal compactness in variational analysis, J. Math. Anal. Appl. 282 (2003), 63-84.

[21] Mordukhovich, B. S. and Wang, B.: Restrictive metric regularity and generalized differential calculus in Banach spaces, Preprint No. 15 (2002), Dept. of Math., Wayne State University, Detroit.

[22] Outrata, J. V.: A generalized mathematical program with equilibrium constraints, SIAM J. Control Optim. 38 (2000), 1623-1638.

[23] Outrata, J. V., Kočvara, M. and Zowe, J.: Nonsmooth Approach to Optimization Problems with Equilibrium Constraints, Kluwer Academic Publishers, Dorderecht, The Netherlands, 1998.

[24] Phelps, R. R.: Convex Functions, Monotone Operators and Differentiability, 2nd edition, Springer, Berlin, 1993.

[25] Rockafellar, R. T. and Wets, R. J-B.: Variational Analysis, Springer, Berlin, 1998.

[26] Thibault, L.: On compactly Lipschitzian mappings, In: P. Gritzmann et al,(eds.), Recent Advances in Optimization, Lecture Notes in Econ. Math. Syst. Ser. 456, Springer, Berlin, 1997, pp. 356-364.

[27] Ye, J. J.: Constraint qualifications and necessary optimality conditions for optimization problems with variational inequality constraints, SIAM J. Optim. 10 (2000), 943-962.

[28] Zhang, R.: Problems of hierarchical optimization in finite dimensions, SIAM J. Optim. 4 (1994), 521536. 